\title{
On Waverider Aerodynamics Experimental Data Analysis Features
}

\author{
D. S. Yatsukhno \\ Ishlinsky Institute for Problems in Mechanics RAS, \\ Moscow, 119526, Russia \\ yatsukhno-ds@rambler.ru
}

\begin{abstract}
The study is devoted to the investigation of the waverider experimental data interpretation. Two features affects the correct comparison opportunity. The first is the virtual and experimental model differences, i.e. the different wetted area. The second is the drag coefficient terms evaluation: pressure drag, skin friction and base drag. The skin friction calculations were performed by the flat plate correlations. The results show that skin friction plays the significant role in the overall drag within the wide Mach number range.
\end{abstract}

Keywords: waveriders, skin friction, base drag, lift-to-drag ratio

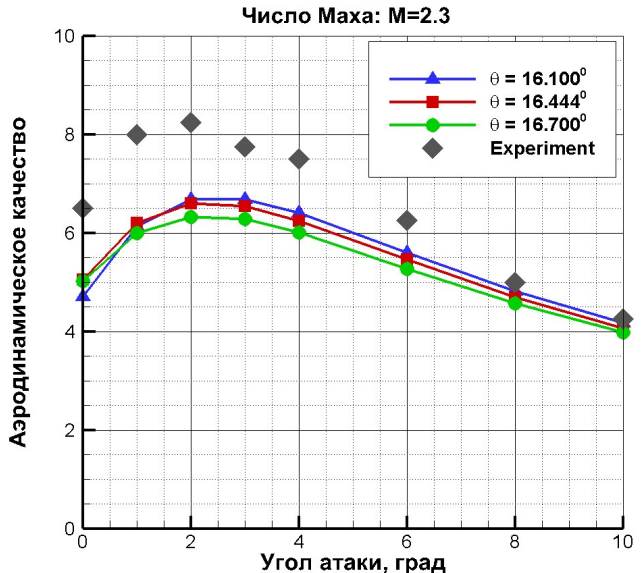

Comparison between the numerical and experimental lift-to-drag ratio for waverider from [22] defined by different cone shock angle at $\mathrm{M}_{\infty}=2.3$ for wide range of the angle of attack

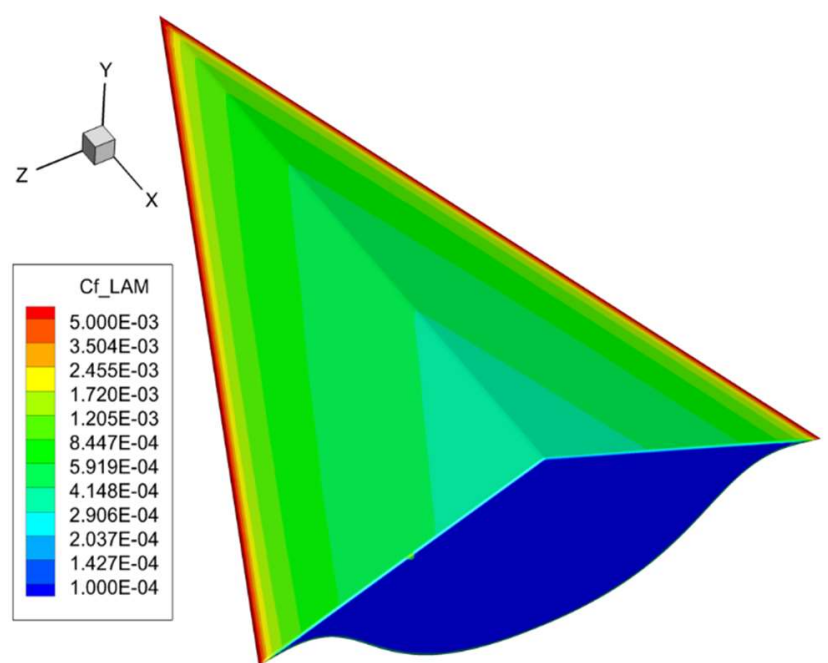

Laminar skin friction coefficient distribution for waverider from $[4-5,20]$ at $\mathrm{M}_{\infty}=4$ and $\alpha=0^{\circ}$

Freestream conditions [22]

\begin{tabular}{|c|c|c|c|}
\hline Parameter & \multicolumn{5}{|l|}{} \\
\hline Pressure $p_{\infty}, \mathrm{erg} / \mathrm{cm}^{3}$ & 55240 & 12400 & 7440 \\
\hline Density $\rho_{\infty}, \mathrm{g} / \mathrm{cm}^{3}$ & $0.122 \times 10^{-3}$ & $0.516 \times 10^{-4}$ & $0.387 \times 10^{-4}$ \\
\hline Viscosity $\mu_{\infty}, \mathrm{g} / \mathrm{cm} \times \mathrm{s}$ & $0.108 \times 10^{-3}$ & $0.580 \times 10^{-4}$ & $0.451 \times 10^{-4}$ \\
\hline Temperature $T_{\infty}, \mathrm{K}$ & 158.1 & 84.3 & 67.1 \\
\hline Mach number $\mathrm{M}_{\infty}$ & 2.3 & 4 & 4.63 \\
\hline
\end{tabular}


УДК533.6.011.5

\title{
О некоторых особенностях анализа экспериментальных данных по аэродинамике волнолетов
}

\author{
Д. С. Яцухно \\ Институт проблем механики им. А.Ю. Иилинского Российской академии наук, \\ Россия, Москва, 119526, проспект Вернадского, д. 101, корп. 1 \\ yatsukhno-ds@rambler.ru
}

\begin{abstract}
Аннотация
Данная статья посвящена исследованию отдельных вопросов интерпретации экспериментальных данных по аэродинамическим характеристикам волнолетов. Среди факторов, непосредственно влияющих на возможность корректного сопоставления результатов расчетов и эксперимента, выделяются два. Во-первых, локальное несовпадение формы виртуальной модели волнолета и образца для стендового испытания, которое заключается в некотором различии площадей смачиваемой поверхности. Во-вторых, структура коэффициента лобового сопротивления исследуемой конфигурации, который включает в себя компоненты, обусловленные давлением, поверхностным трением и сопротивлением донного среза. Расчет поверхностного трения осуществлялся с использованием приближенной методики, основанной на определении локального коэффициента трения по соотношениям для плоской пластины как в ламинарном, так и турбулентном случае. Результаты расчетов показывают, что учет поверхностного трения качественно меняет характер распределения коэффициента лобового сопротивления при различных числах Маха.
\end{abstract}

Ключевые слова: волнолеты, поверхностное трение, донное сопротивление, аэродинамическое качество

\section{1. Введение}

В работах [1-2] был рассмотрен класс волнолетов, форма которых была затронута процедурой так называемой вязкостной оптимизации. Она позволила повысить суммарное аэродинамическое качество и выйти за пределы асимптотической кривой, называемой барьером Кюхемана [3]. В ходе исследования была также переосмыслена теоретическая концепция волнолета и доказана необходимость учета поверхностного трения как в процессе вычисления координат точек поверхности, так и при расчете аэродинамического качества. Подходы к оценке вязкого сопротивления могут быть различными. В статьях [4-5] коэффициент трения был получен путем численного интегрирования уравнений Навье-Стокса, в том числе параболизованных, и вычисления компонент тензора напряжений. В работах [1] и [6]суть приближенного метода состоит в интегрировании обыкновенных дифференциальных уравнений импульса и энергии вдоль линий тока на границе пограничного слоя. Для ламинарного пограничного слоя используется метод Вальца [7], в турбулентном случае применяется метод Уайта и Кристофа [8]. Переходный режим течения анализируется с помощью эмпирических соотношений теории пограничного слоя на плоской пластине[9-10]. В статьях $[2,11]$ трение рассчитывается при помощи метода характерной температуры [12]. Все перечисленные методики существенно различаются по сложности реализации и вычислительным затратам, поэтому следует учесть их взаимную погрешность расчета сопротивления трения. В работе [2] было отмечено, что различие между наиболее простым методом характерной температуры и 
более сложными подходами, использующими соотношения теории пограничного слоя, составляет примерно 15 \%. Это дает возможность использовать приближенные методы в рамках настоящей работы.

\section{2. Постановка задачи}

Компьютерный код UST3D-AUSM-ECUSP [13] является развитием компьютерного кода UST3D [14-18] в части приближенного решения задачи Римана, которое основывается на использовании AUSM-ECUSP схемы [19]. Интегрирование полной системы уравнений Навье-Стокса позволяет достаточно точно определить газодинамические параметры как в ближнем поле течения, так и по поверхности исследуемой модели. В то же время возможности исследования вязких эффектов существенно ограничены используемыми в расчетах неструктурированными тетраэдральными сетками. Векторная форма газодинамических уравнений имеет вид

$$
\frac{\partial \mathbf{w}}{\partial t}+\frac{\partial}{\partial x}\left(\mathbf{F}_{x}\right)+\frac{\partial}{\partial y}\left(\mathbf{F}_{y}\right)+\frac{\partial}{\partial z}\left(\mathbf{F}_{z}\right)=\frac{\partial}{\partial x}\left(\mathbf{G}_{x}\right)+\frac{\partial}{\partial y}\left(\mathbf{G}_{y}\right)+\frac{\partial}{\partial z}\left(\mathbf{G}_{z}\right)
$$

В состав компонент вектора консервативных переменных $\mathbf{w}$ входят следующие величины: $\rho$ - плотность; $u, v, w$ - проекции вектора скорости; $E$ - удельная полная энергия. Вектор имеет вид

$$
\mathbf{w}=\left[\begin{array}{lllll}
\rho & \rho u & \rho v & \rho w & \rho E
\end{array}\right]^{\mathrm{T}}
$$

Компоненты вектора невязкого потока $\mathbf{F}_{x}, \mathbf{F}_{y}$ и $\mathbf{F}_{z}$ могут быть представлены в виде

$$
\mathbf{F}_{x}=\left[\begin{array}{l}
\rho u \\
\rho u^{2}+p \\
\rho u v \\
\rho u w \\
(\rho E+p) u
\end{array}\right], \quad \mathbf{F}_{y}=\left[\begin{array}{l}
\rho v \\
\rho u v \\
\rho v^{2}+p \\
\rho v w \\
(\rho E+p) v
\end{array}\right] \quad \mathbf{F}_{z}=\left[\begin{array}{l}
\rho w \\
\rho w u \\
\rho w v \\
\rho w^{2}+p \\
(\rho E+p) w
\end{array}\right]
$$

Здесь $p$-давление. Вектор невязкого потока содержит в себе две физически различных компоненты: конвективную и зависящую от давления. В отношении последней для приближенного решения задачи о распаде произвольного разрыва используются соотношения расщепления в соответствии с идеями AUSM-ECUSP схемы [19]

$$
\begin{gathered}
p=p_{L}^{+}+p_{R}^{-}, \\
p V=(p V)_{L}^{+}+(p V)_{R}^{-}
\end{gathered}
$$

Слагаемые в уравнении (4) - определяются следующим образом

$$
p_{L, R}^{ \pm}=\left\{\begin{array}{lll}
\frac{p}{2} \frac{(\mathrm{M} \pm|\mathrm{M}|)}{\mathrm{M}}, & \text { при } & |\mathrm{M}|>1, \\
\frac{p}{4}(\mathrm{M} \pm 1)^{2}(2 \mp \mathrm{M}), & \text { при } & |\mathrm{M}| \leq 1
\end{array}\right.
$$

Здесь М - число Маха. Аналогично для уравнения (5)

$$
(p V)_{L, R}^{ \pm}= \begin{cases}\frac{p V_{N, K}^{\text {norm }}}{2} \frac{(\mathrm{M} \pm|\mathrm{M}|)}{\mathrm{M}}, & \text { при }|\mathrm{M}|>1, \\ \frac{p}{2}\left(V_{N, K}^{\text {norm }} \pm \mathrm{a}_{1 / 2}\right), & \text { при }|\mathrm{M}| \leq 1\end{cases}
$$


Здесь $V_{N, K}^{\text {norm }}$ - нормальная к грани $K$ элемента $N$ составляющая вектора скорости; $\mathrm{a}_{1 / 2}-$ среднее арифметическое значение скорости звука между соседствующими через определенную грань тетраэдральными элементами.

При расчете конвективных эффектов также выполняется расщепление числа Маха

$$
\begin{gathered}
\mathrm{M}_{1 / 2}=\mathrm{M}_{L}^{+}+\mathrm{M}_{R}^{-}, \\
\mathrm{M}_{L, R}^{ \pm}=\left\{\begin{array}{l}
\frac{1}{2}(\mathrm{M} \pm|\mathrm{M}|), \text { при }|\mathrm{M}|>1, \\
\pm \frac{1}{4}(\mathrm{M} \pm 1)^{2}, \text { при }|\mathrm{M}| \leq 1
\end{array}\right.
\end{gathered}
$$

Вязкие члены $\mathbf{G}_{x}, \mathbf{G}_{y}$ и $\mathbf{G}_{z}$ определяются в соответствии с выражениями

$$
\mathbf{G}_{x}=\left[\begin{array}{c}
0 \\
\tau_{x x} \\
\tau_{x y} \\
\tau_{x z} \\
u \tau_{x x}+v \tau_{x y}+w \tau_{x z}-q_{x}
\end{array}\right], \mathbf{G}_{y}=\left[\begin{array}{c}
0 \\
\tau_{y x} \\
\tau_{y y} \\
\tau_{y z} \\
u \tau_{y x}+v \tau_{y y}+w \tau_{y z}-q_{y}
\end{array}\right], \mathbf{G}_{z}=\left[\begin{array}{c}
0 \\
\tau_{z x} \\
\tau_{z y} \\
\tau_{z z} \\
u \tau_{z x}+v \tau_{z y}+w \tau_{z z}-q_{z}
\end{array}\right]
$$

В уравнении (10) $q_{x}, q_{y}, q_{z}$ - составляющие вектора теплового потока. Компоненты тензора вязких напряжений определяются по формулам

$$
\begin{aligned}
& \tau_{x x}=\mu\left(\frac{4}{3} \frac{\partial u}{\partial x}-\frac{2}{3} \frac{\partial v}{\partial y}-\frac{2}{3} \frac{\partial w}{\partial z}\right), \tau_{y y}=\mu\left(\frac{4}{3} \frac{\partial v}{\partial y}-\frac{2}{3} \frac{\partial u}{\partial x}-\frac{2}{3} \frac{\partial w}{\partial z}\right) \\
& \tau_{z z}=\mu\left(\frac{4}{3} \frac{\partial w}{\partial z}-\frac{2}{3} \frac{\partial u}{\partial x}-\frac{2}{3} \frac{\partial v}{\partial y}\right), \tau_{x y}=\tau_{y x}=\mu\left(\frac{\partial v}{\partial x}+\frac{\partial u}{\partial y}\right) \\
& \tau_{y z}=\tau_{z y}=\mu\left(\frac{\partial w}{\partial y}+\frac{\partial v}{\partial z}\right), \tau_{x z}=\tau_{z x}=\mu\left(\frac{\partial u}{\partial z}+\frac{\partial w}{\partial x}\right)
\end{aligned}
$$

Здесь $\mu$ - коэффициент динамической вязкости. Для замыкания системы уравнений газовой динамики используются: термическое и калорическое уравнения состояния, закон Фурье

$$
\begin{gathered}
p=(\gamma-1) \rho U=(\gamma-1) \rho\left[E-0.5\left(u^{2}+v^{2}+w^{2}\right)\right], \\
U=c_{V} T, \\
q=-\lambda \nabla T
\end{gathered}
$$

Связь между кинетической и полной энергией определяется с помощью выражением

$$
E=\frac{p}{(\gamma-1) \rho}+\frac{u^{2}+v^{2}+w^{2}}{2}
$$

Здесь $\gamma$ - показатель адиабаты; $U$ - удельная внутренняя энергия; $\lambda$ - коэффициент теплопроводности; $T$ - температура; $c_{V}$ - удельная теплоемкость при постоянном объеме. 


\section{3. Результаты численного моделирования}

На первом этапе проводилась верификация приближенного метода оценки поверхностного трения для конфигурации ЭК-волнолета (рис. 1). Данная модель уже рассматривалась в ряде работ теоретического [4-5, 13] и экспериментального [20] характера. Наибольший интерес состоит в сравнении результатов настоящей работы и решений уравнений НавьеСтокса с учетом зоны вязкого пограничного слоя [4-5].

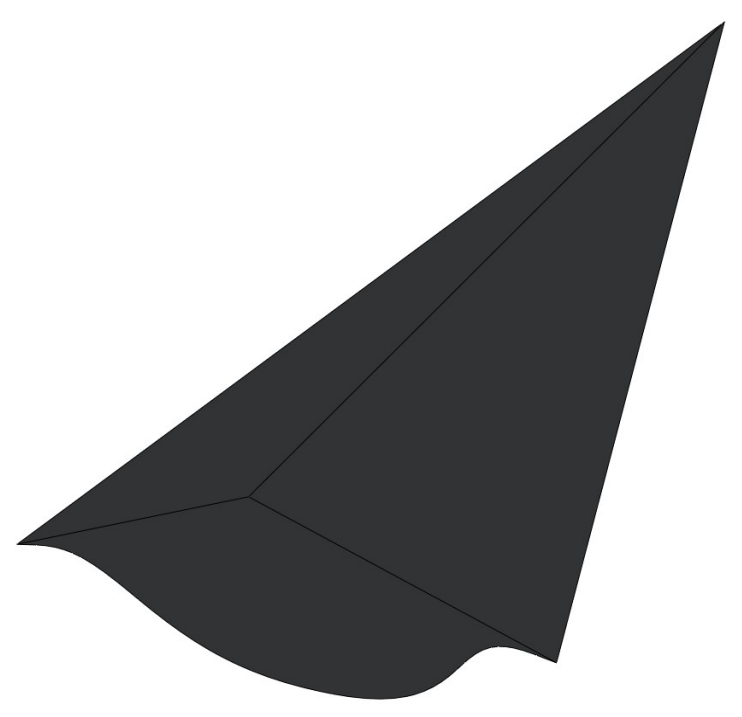

Рис. 1. Виртуальная модель ЭК-Волнолета

Исследуемый волнолет имеет острые кромки, что предъявляет определенные требования к определению линий тока на триангулированной поверхности аппарата. В отличие от затупленного тела, которое имеет критическую точку, из которой в свою очередь исходят линии тока, в данном случае через каждую точку передней кромки будет проходить линия тока. С учетом этого имеет смысл выделить зону «стартовых» треугольников вдоль кромки, которая будет характеризоваться повышенными значениями поверхностного трения. Суть используемого приближенного метода заключается в расчете местного коэффициента трения вдоль линии тока, используя соотношения для плоской пластины $[8,21]$

$$
\begin{aligned}
c_{f}^{\text {lam }} & =\frac{0.664}{\operatorname{Re}_{x}^{0.5}}\left(\frac{p}{p_{\infty}}\right)^{0.5}\left(\frac{\mu^{*}}{\mu_{\infty}} \frac{T_{\infty}}{T^{*}}\right)^{0.5}, \\
c_{f}^{\text {turb }} & =\frac{0.0592}{\operatorname{Re}_{x}^{0.2}}\left(\frac{p}{p_{\infty}}\right)^{0.2}\left(\frac{\mu^{*}}{\mu_{\infty}} \frac{T_{\infty}}{T^{*}}\right)^{0.2}
\end{aligned}
$$

В соотношениях (16) - (17) $p_{\infty}, T_{\infty}, \mu_{\infty}$ - давление, температура, вязкость в набегающем потоке; $p$ - местное давление; $T^{*}$ - характерная температура; $\mu^{*}$ - вязкость при характерной температуре. Число Рейнольдса определяется в соответствии с длиной каждой линии тока, а плотность, скорость и вязкость соответствуют набегающему потоку

$$
\operatorname{Re}_{x}=\frac{\rho_{\infty} V_{\infty} x}{\mu_{\infty}}
$$

Здесь $x$ - длина линии тока. Характерная температура вычисляется по формуле Эккерта

$$
T^{*}=T_{\infty}\left[1+0.032 \mathrm{M}_{\infty}^{2}+0.58\left(\frac{T_{w}}{T_{\infty}}-1\right)\right]
$$


Здесь $T_{w}$ - температура стенки; $\mathrm{M}_{\infty}$ - число Маха набегающего потока. В соответствии с формулами (16)-(19) коэффициент поверхностного трения зависит от местного давления и длины линии тока. В табл. 1-2 представлены значения параметров невозмущенного течения и характерной площади для расчета аэродинамических коэффициентов.

\section{Таблица 1}

Параметры набегающего потока для ЭК-волнолета

\begin{tabular}{|c|c|}
\hline Параметр & Значение \\
\hline Давление $p_{\infty}$, эрг $/ \mathrm{cm}^{3}$ & 14530 \\
\hline Плотность $\rho_{\infty}, \Gamma / \mathrm{cm}^{3}$ & $0.175 \times 10^{-4}$ \\
\hline Температура $T_{\infty}, \mathrm{K}$ & 288.9 \\
\hline Число Маха $\mathrm{M}_{\infty}$ & 4 \\
\hline Характерная площадь $S_{r e f}, \mathrm{~cm}^{2}$ & 489 \\
\hline
\end{tabular}

Расчеты выполнялись в две стадии. Сначала проводилось численное моделирование обтекания полных моделей волнолетов с целью определения газодинамических параметров на поверхности, а затем применялся приближенный метод для вычисления вязкого сопротивления. Расчетная сетка включала 3 млн. тетраэдральных элементов.

Результаты расчета поверхностного трения для ЭК-волнолета при углах атаки $\alpha=0^{\circ}$ и $\alpha=5^{\circ}$ показаны на рис. 3-10. Коэффициент трения на нижней поверхности несколько выше, чем на верхней. При увеличении угла атаки за счет разрежения потока снижается вязкое сопротивление на верхней поверхности. Интегральный коэффициент трения $\left(c_{f}^{\text {lam }}=0.00788\right.$, $c_{f}^{\text {turb }}=0.03025$ при $\left.\alpha=0^{\circ}\right)$ также уменьшается с возрастанием угла атаки $\left(c_{f}^{\text {lam }}=0.00747\right.$, $c_{f}^{t u r b}=0.02947$ при $\alpha=5^{\circ}$ ). Таким образом, поверхностное трение верхней части волнолета падает быстрее, чем растет трение на нижней части. Полученные результаты находятся в хорошем соответствии с данными работы [4] ( $c_{f}^{\text {lam }}=0.00737$ при $\alpha=0^{\circ}$ и $c_{f}^{\text {lam }}=0.00672$ при $\left.\alpha=5^{\circ}\right)$.

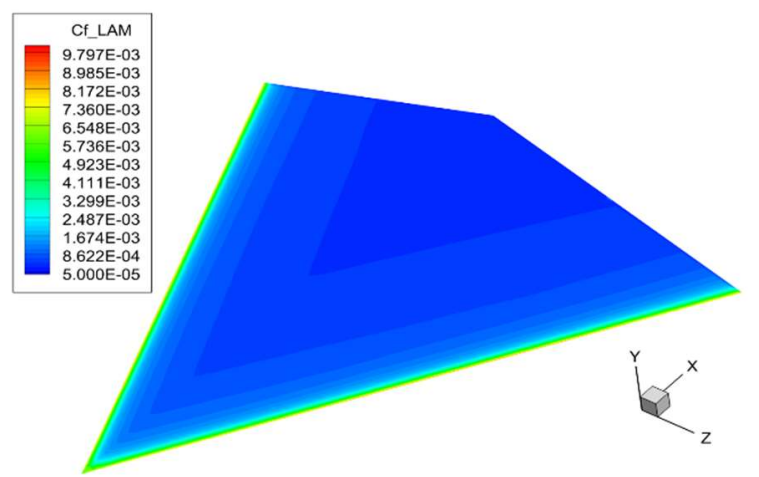

Рис. 3. Коэффициент поверхностного трения для верхней поверхности ЭК-волнолета. Ламинарный случай. Угол атаки $\alpha=0^{\circ}$

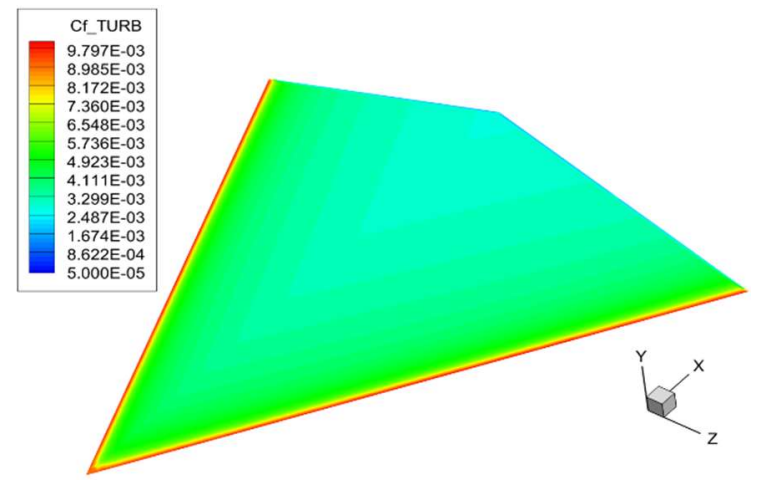

Рис. 4. Коэффициент поверхностного трения для верхней поверхности ЭК-волнолета. Турбулентный случай. Угол атаки $\alpha=0^{\circ}$ 


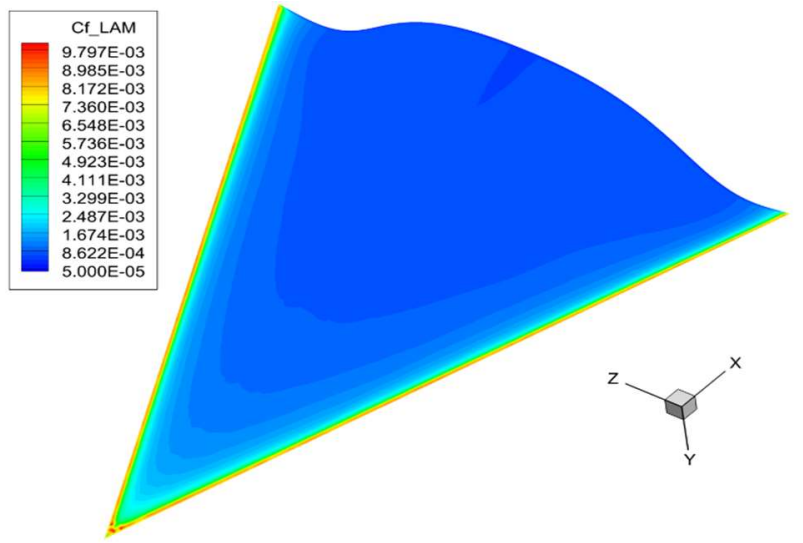

Рис. 5. Коэффициент поверхностного трения для нижней поверхности ЭК-волнолета. Ламинарный случай. Угол атаки $\alpha=0^{\circ}$

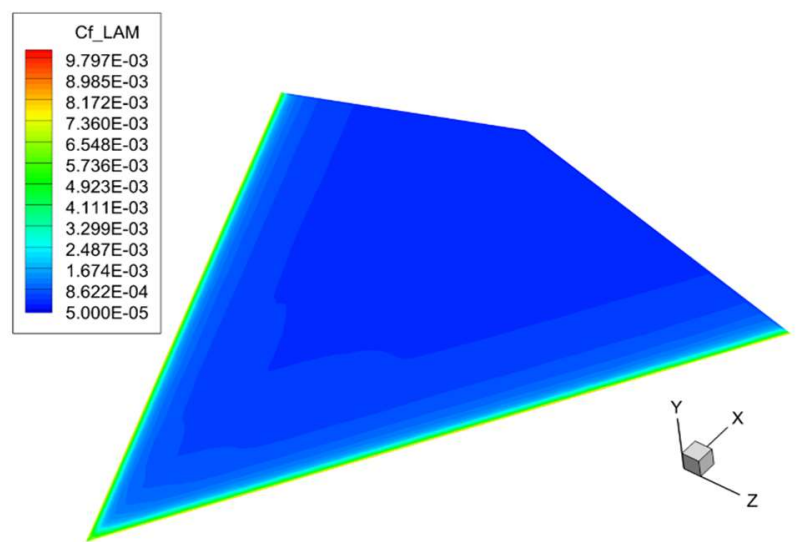

Рис. 7. Коэффициент поверхностного трения для верхней поверхности ЭК-волнолета. Ламинарный случай. Угол атаки $\alpha=5^{\circ}$

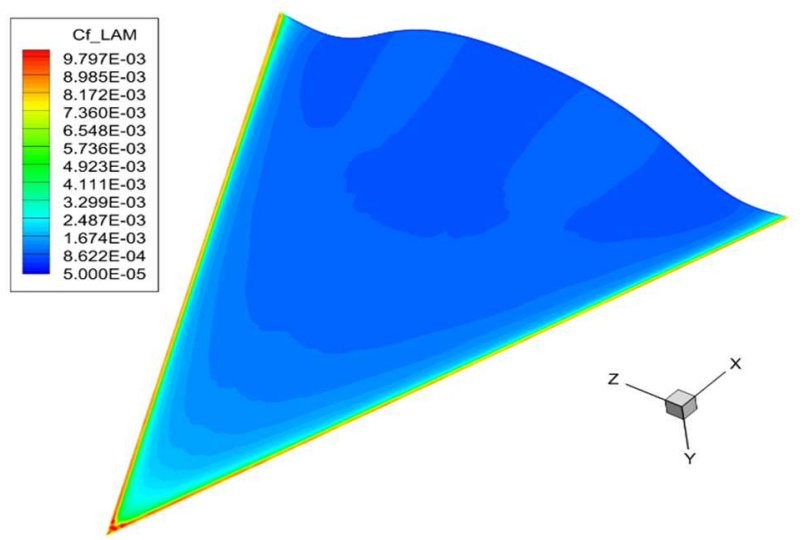

Рис. 9. Коэффициент поверхностного трения для нижней поверхности ЭК-волнолета. Ламинарный случай. Угол атаки $\alpha=5^{\circ}$

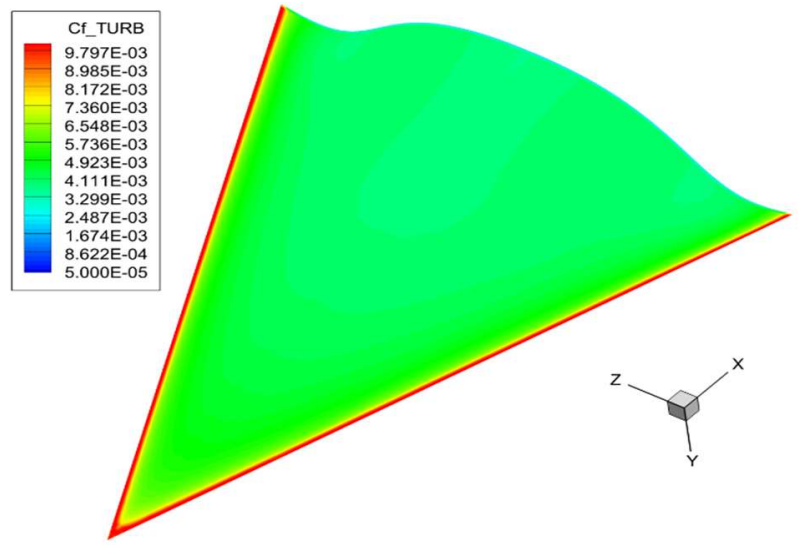

Рис. 6. Коэффициент поверхностного трения для нижней поверхности ЭК-волнолета. Турбулентный случай. Угол атаки $\alpha=0^{\circ}$

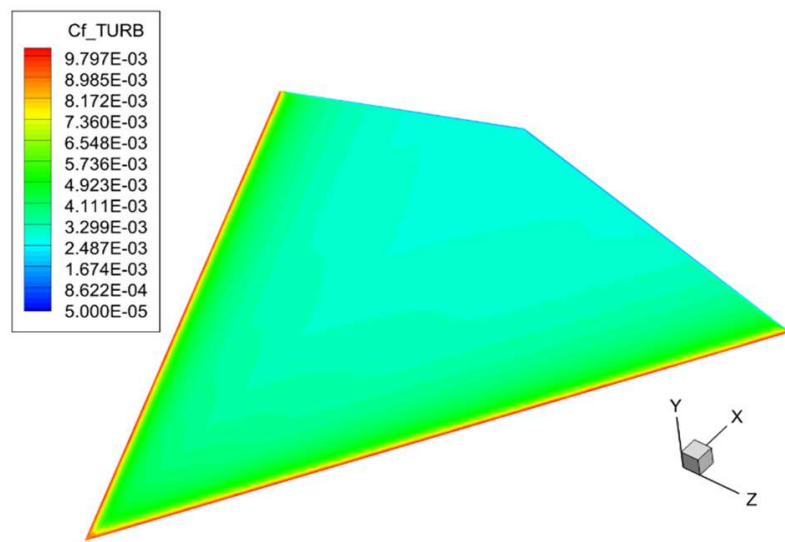

Рис. 8. Коэффициент поверхностного трения для верхней поверхности ЭК-волнолета. Турбулентный случай. Угол атаки $\alpha=5^{\circ}$

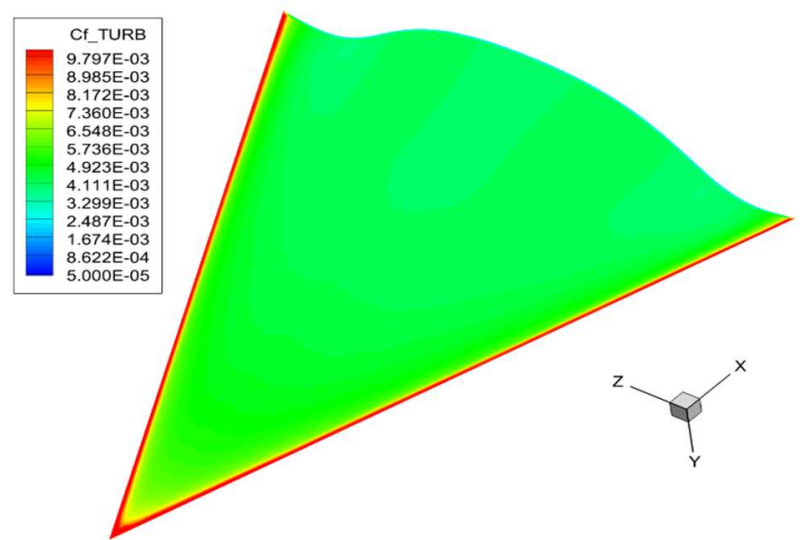

Рис. 10. Коэффициент поверхностного трения для нижней поверхности ЭК-волнолета. Турбулентный случай. Угол атаки $\alpha=5^{\circ}$

На рис. 11-12 показаны распределения коэффициента поверхностного трения в сечении плоскости симметрии (рис. 11) и в поперечном сечении на расстоянии 39.878 см от носа волнолета (рис. 12). В сравнении с результатами расчета по параболизованным уравнениям Навье - Стокса [5] следует отметить монотонный характер изменения вязкого сопротивления в 
поперечном сечении волнолета при использовании приближенной методики. При этом определенная немонотонность в распределении коэффициента трения наблюдается ближе к задней стенке исследуемой конфигурации, что хорошо заметно при увеличении угла атаки (рис. 10).

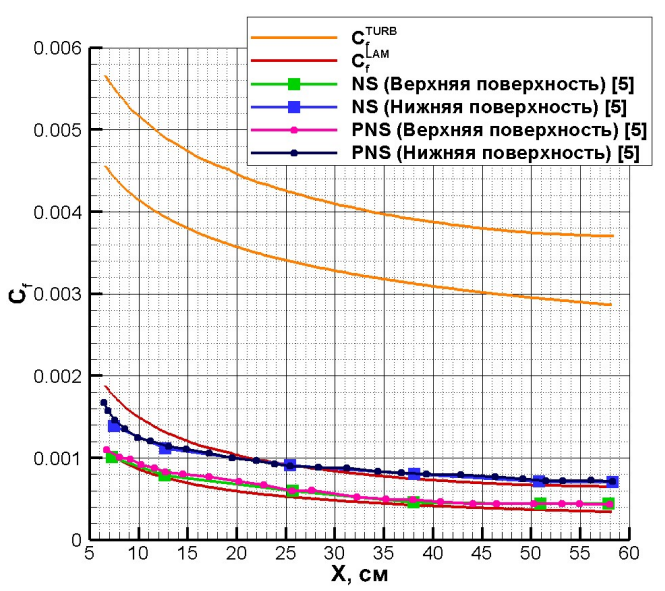

Рис. 11. Коэффициент поверхностного трения в сечении плоскости симметрии ЭК-волнолета. Угол атаки $\alpha=0^{\circ}$

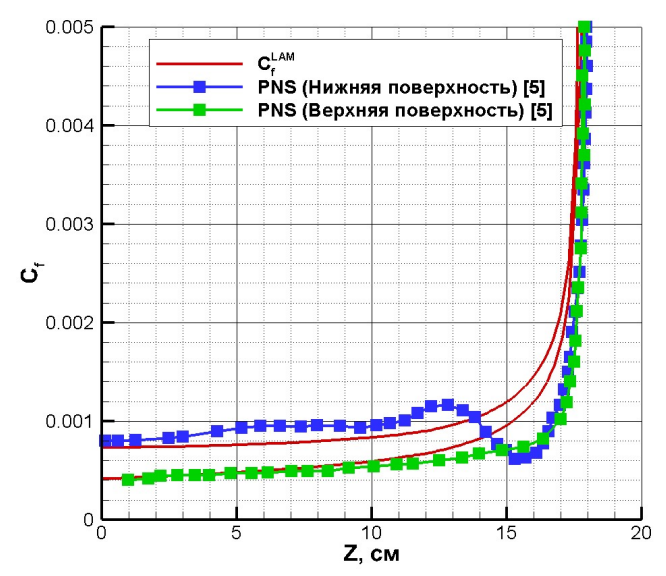

Рис. 12. Коэффициент поверхностного трения в поперечном сечении ЭК-волнолета ( $x=39.878$ см). Угол атаки $\alpha=0^{\circ}$

Второй этап исследования состоял в оценке поверхностного трения для волнолета, построенного на поле течения за прямым конусом (ПК-волнолет). Эта конфигурация уже была исследована с точки зрения оценки аэродинамических характеристик, в том числе и поверхностного трения [16], которое определялось в соответствии с методом характерной температуры [11], в то время как газодинамические расчеты были выполнены с использованием компьютерного кода UST3D [14]. В данной работе применяются соотношения (16)-(19), которые обеспечивают корректное относительное распределение коэффициента трения по верхней и нижней частям исследуемой формы. На рис. 13 показана модель ПК-волнолета, для которой в работе [22] представлены экспериментальные данные по аэродинамическим характеристикам для чисел Маха $\mathrm{M}_{\infty}=2.3, \mathrm{M}_{\infty}=4$ и $\mathrm{M}_{\infty}=4.63$. При сравнении результатов расчетов с экспериментальными данными следует учитывать возможные несоответствия геометрии виртуальной модели и экспериментального образца. Поэтому было выполнено численное моделирование обтекания трех моделей ПК-волнолета, которые отличались углом полураствора исходной конической ударной волны $\left(\theta=16.1^{\circ}, \theta=16.444^{\circ}\right.$ и $\left.\theta=16.7^{\circ}\right)$. На рис. $14-15$ показаны различия между подобными моделями ПК-волнолета. При расчетах использовались сетки, включающие от 2 до 3 млн. тетраэдральных элементов.

В таблице 2 представлены параметры набегающего потока для разных чисел Маха. Все исходные данные соответствуют размерному числу Рейнольдса $\mathrm{Re}=6.56 \times 10^{4} \mathrm{1} / \mathrm{cm}$.

\section{Таблицุа 2}

\section{Параметры набегающего потока [22]}

\begin{tabular}{|c|c|c|c|}
\hline Параметр & \multicolumn{5}{|c|}{} \\
\cline { 1 - 3 } Давление $p_{\infty}$, эрг $/ \mathrm{cm}^{3}$ & 55240 & 12400 & 7440 \\
\hline Плотность $\rho_{\infty}$, г $/ \mathrm{cm}^{3}$ & $0.122 \times 10^{-3}$ & $0.516 \times 10^{-4}$ & $0.387 \times 10^{-4}$ \\
\hline Вязкость $\mu_{\infty}, \Gamma / \mathrm{cm} \times \mathrm{c}^{-3}$ & $0.108 \times 10^{-3}$ & $0.580 \times 10^{-4}$ & $0.451 \times 10^{-4}$ \\
\hline Температура $T_{\infty}, \mathrm{K}$ & 158.1 & 84.3 & 67.1 \\
\hline Число Маха $\mathrm{M}_{\infty}$ & 2.3 & 4 & 4.63 \\
\hline
\end{tabular}




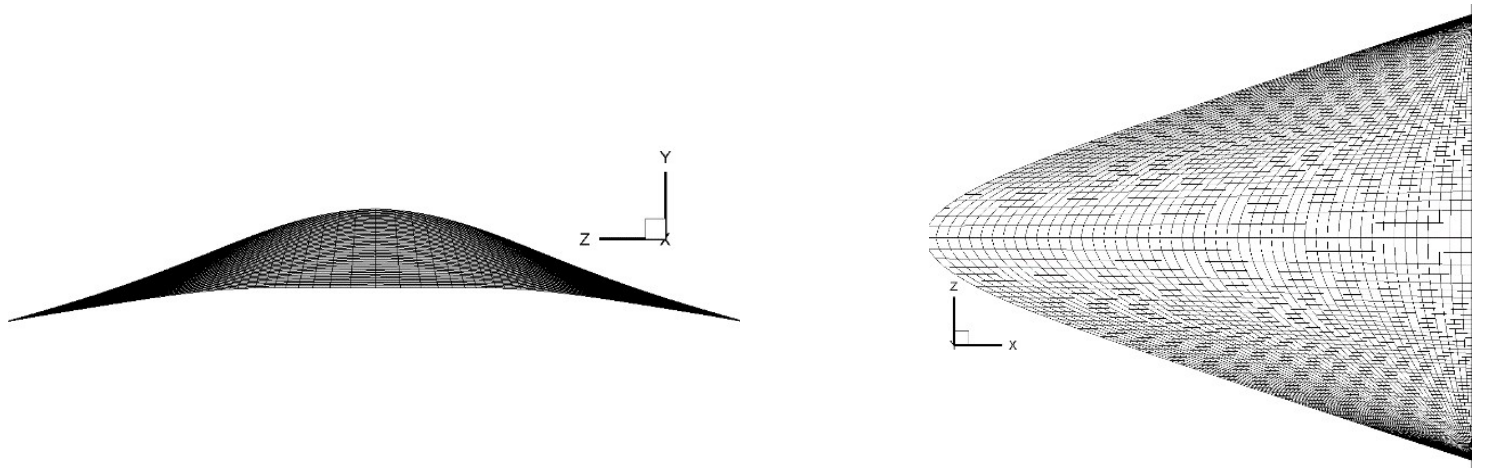

(a)

(б)

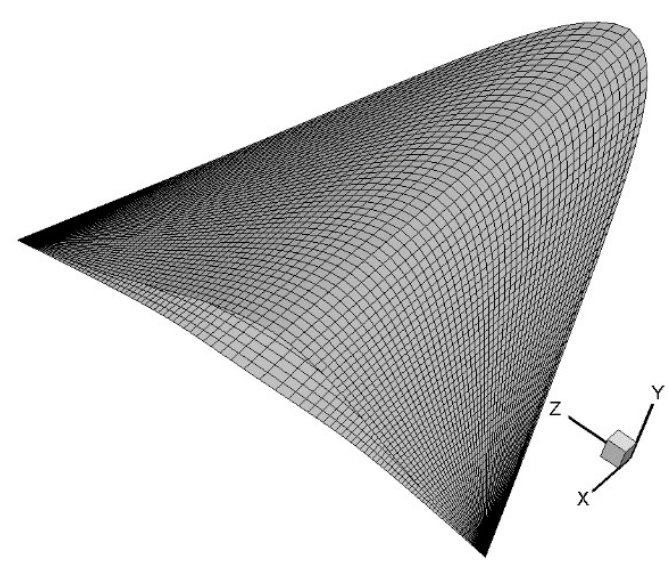

(в)

Рис. 13. Виртуальная модель ПК-волнолета: (a) вид сзади, (б) вид сверху и (в) перспектива

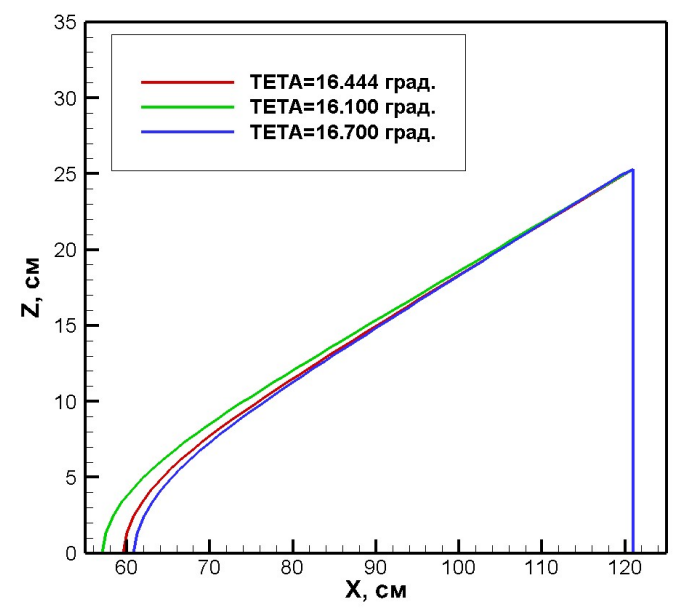

Рис. 14. Горизонтальная проекция моделей ПКволнолета, построенных на конических ударных волнах с различным углом полураствора

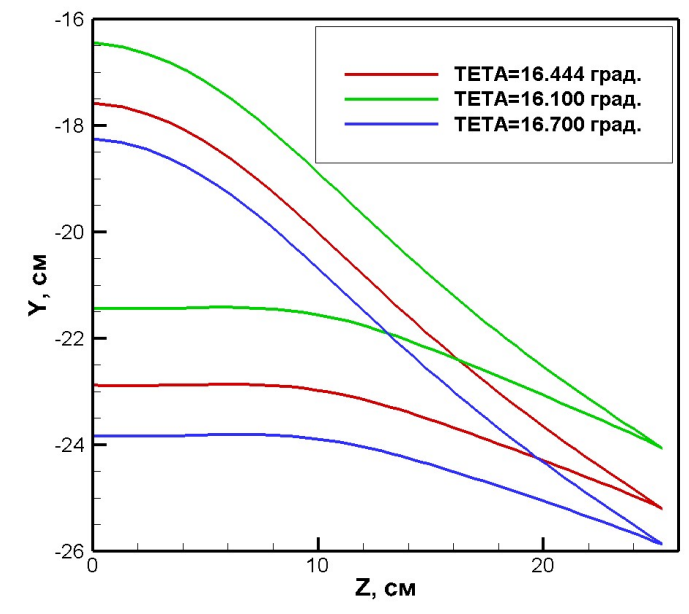

Рис. 15. Фронтальная проекция моделей ПКволнолета, построенных на конических ударных волнах с различным углом полураствора

Результаты систематических расчетов аэродинамических характеристик трех моделей ПК-волнолета представлены на рис. 16-39. Для каждой геометрии расчетные данные структурированы следующим образом: коэффициент подъемной силы в зависимости от числа Маха набегающего потока и угла атаки (рис. 16, 24, 32), коэффициент лобового сопротивления при учете только действия сил давления в зависимости от числа Маха набегающего по- 
тока и угла атаки (рис. 17, 25, 33), коэффициент лобового сопротивления, включающий донное сопротивление, в зависимости от числа Маха набегающего потока и угла атаки (рис. 18, $26,34)$, ламинарный коэффициент поверхностного трения в зависимости от числа Маха набегающего потока и угла атаки (рис. 19, 27, 35), турбулентный коэффициент поверхностного трения в зависимости от числа Маха набегающего потока и угла атаки (рис. 20, 28, 36), сравнительный анализ компонент коэффициента сопротивления при разных углах атаки для числа Маха $\mathrm{M}_{\infty}=2.3$ (рис. 21, 29, 37), $\mathrm{M}_{\infty}=4$ (рис. 22, 30, 38), $\mathrm{M}_{\infty}=4.63$ (рис. 23, 31, 39). За исключением коэффициента сопротивления давления, прочие составляющие лобового сопротивления слабо зависят от угла атаки. Сопротивление донного среза при малых углах атаки является основной компонентой общего сопротивления, причем чем меньше число Маха, тем больше максимальный угол атаки при котором донное сопротивление превышает сопротивление от действия сил давления.

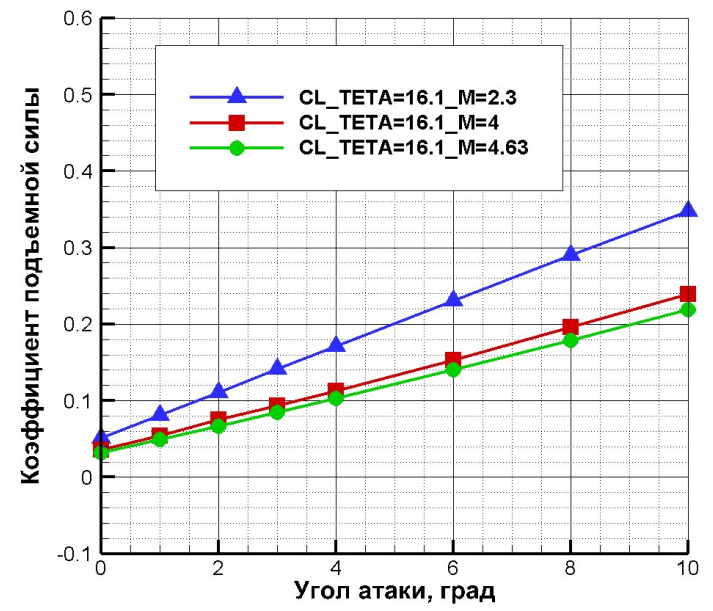

Рис. 16. Коэффициент подъемной силы для разных чисел Маха при различных углах атаки. $\theta=16.1^{\circ}$

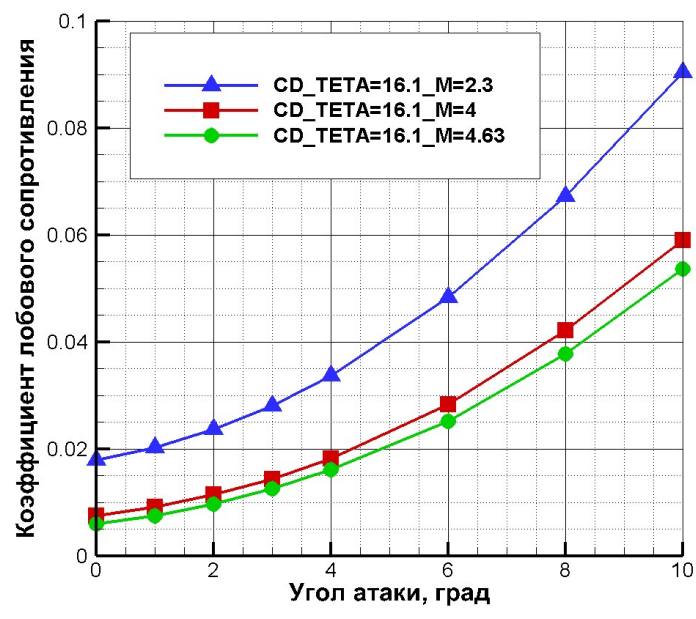

Рис. 18. Коэффициент лобового сопротивления с учетом донного сопротивления для разных чисел Маха при различных углах атаки. $\theta=16.1^{\circ}$

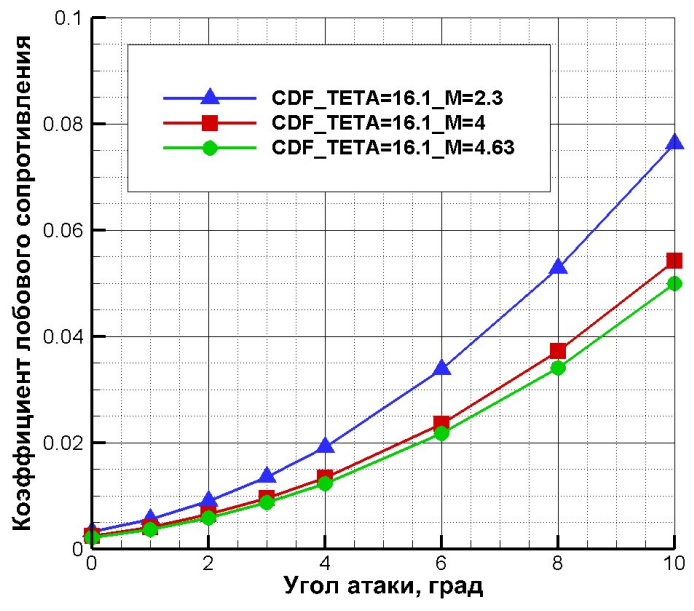

Рис. 17. Коэффициент лобового сопротивления без учета донного сопротивления для разных чисел Маха при различных углах атаки. $\theta=16.1^{\circ}$

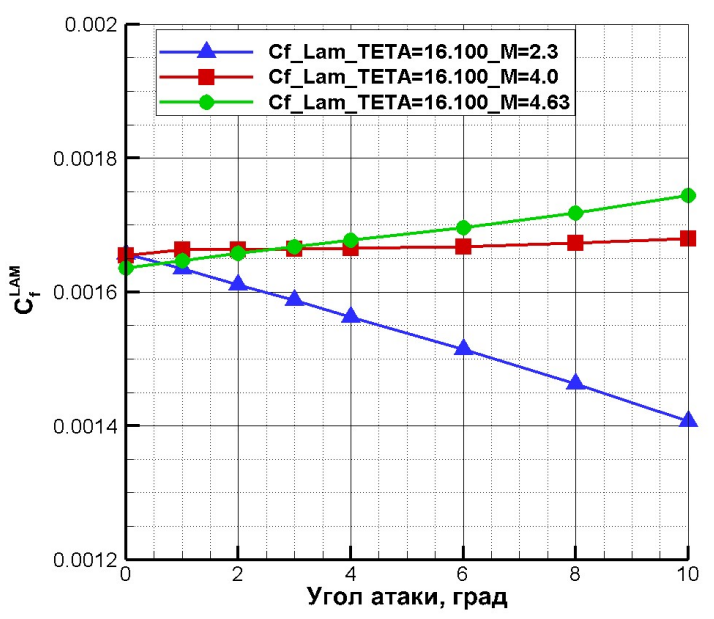

Рис. 19. Коэффициент поверхностного трения для разных чисел Маха при различных углах атаки. Ламинарный случай. $\theta=16.1^{\circ}$ 


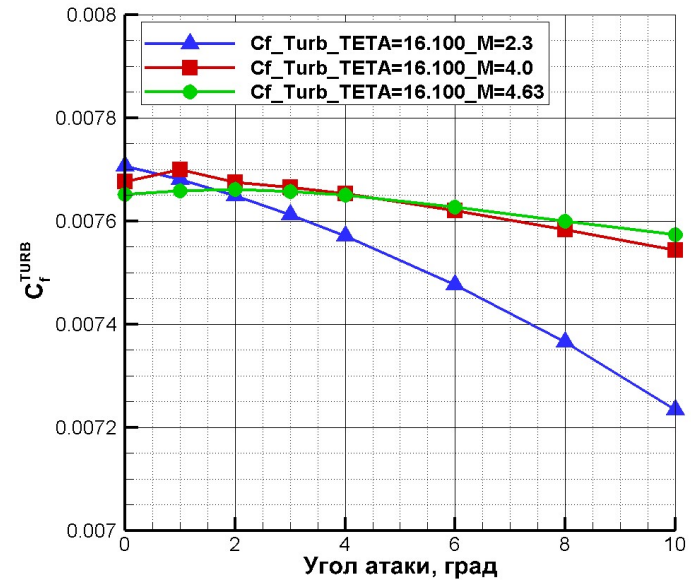

Рис. 20. Коэффициент поверхностного трения для разных чисел Маха при различных углах атаки. Турбулентный случай. $\theta=16.1^{\circ}$

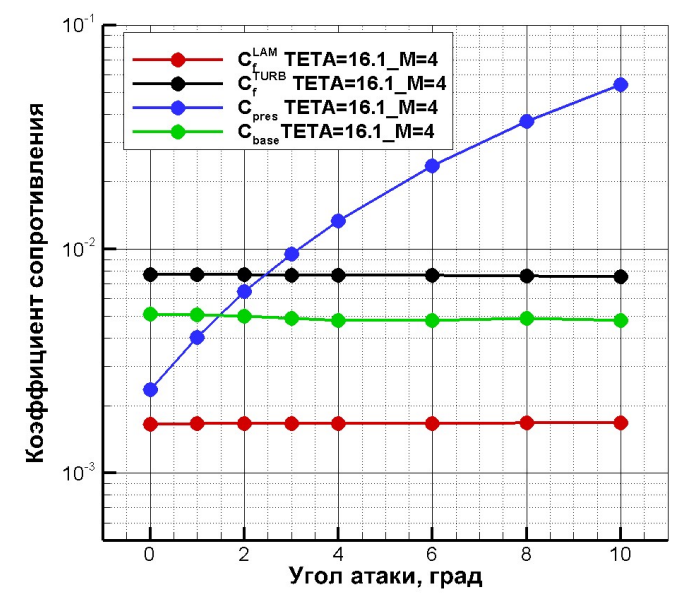

Рис. 22. Компоненты сопротивления волнолета при различных углах атаки. $\mathrm{M}_{\infty}=4, \theta=16.1^{\circ}$

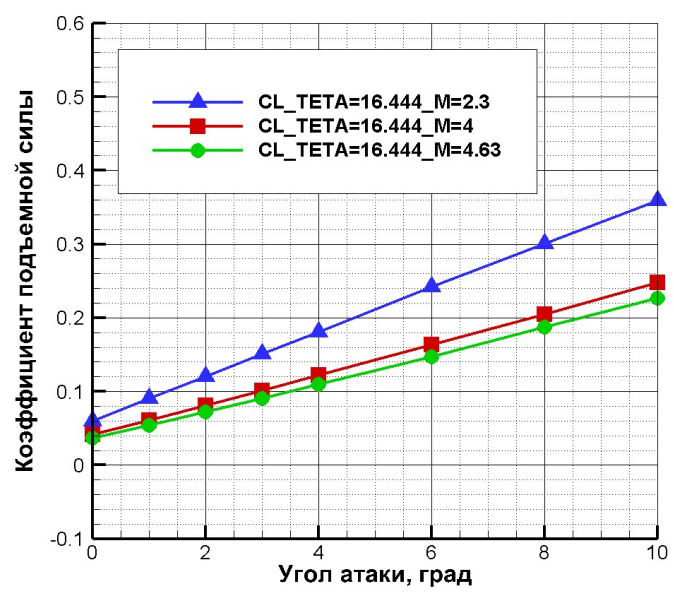

Рис. 24. Коэффициент подъемной силы для разных чисел Маха при различных углах атаки. $\theta=16.444^{\circ}$

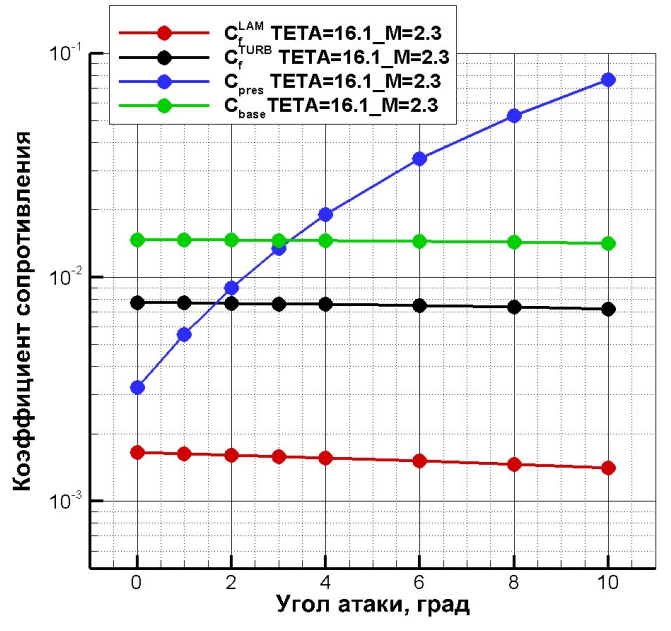

Рис. 21. Компоненты сопротивления ПК-волнолета при различных углах атаки. $\mathrm{M}_{\infty}=2.3$, $\theta=16.1^{\circ}$

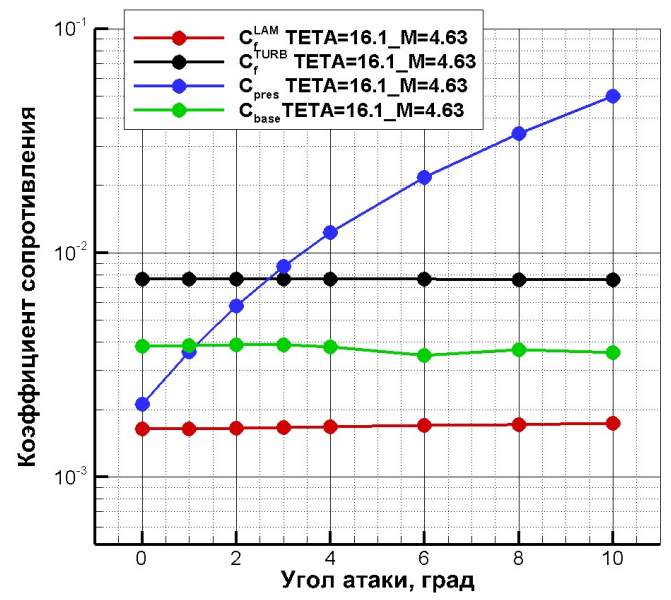

Рис. 23. Компоненты сопротивления ПК-волнолета при различных углах атаки. $\mathrm{M}_{\infty}=4.63$, $\theta=16.1^{\circ}$

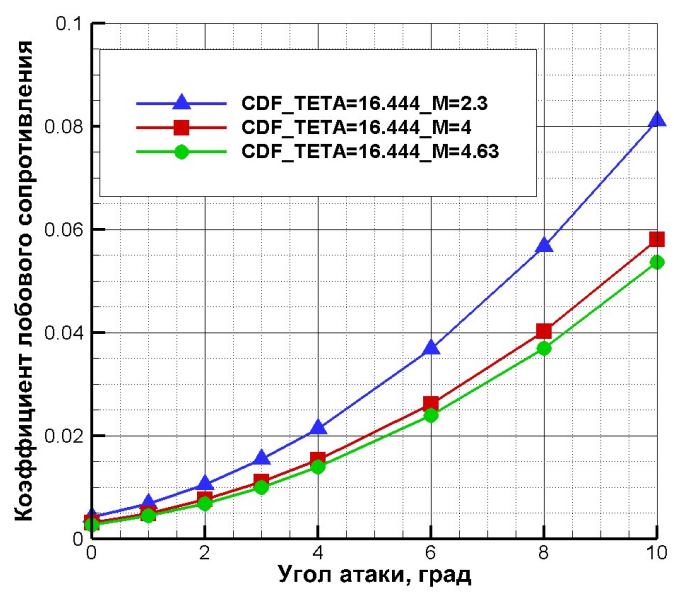

Рис. 25. Коэффициент лобового сопротивления без учета донного сопротивления для разных чисел Маха при различных углах атаки. $\theta=16.444^{\circ}$ 


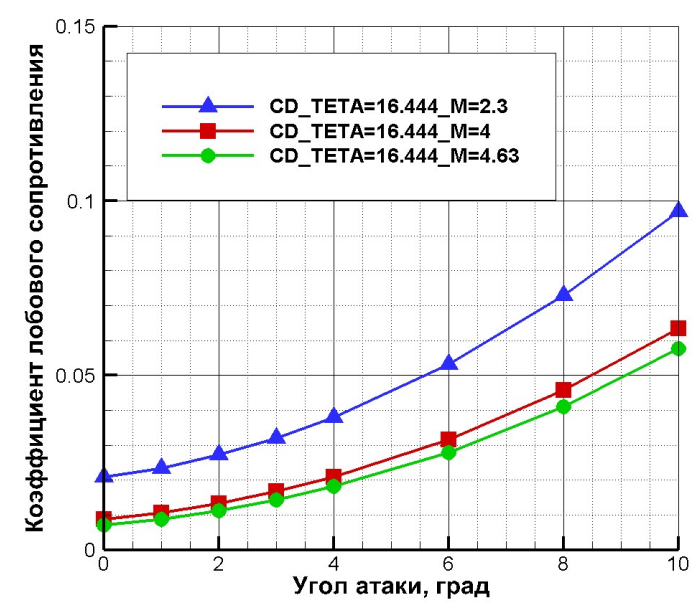

Рис. 26. Коэффициент лобового сопротивления с учетом донного сопротивления для разных чисел Маха при различных углах атаки. $\theta=16.444^{\circ}$

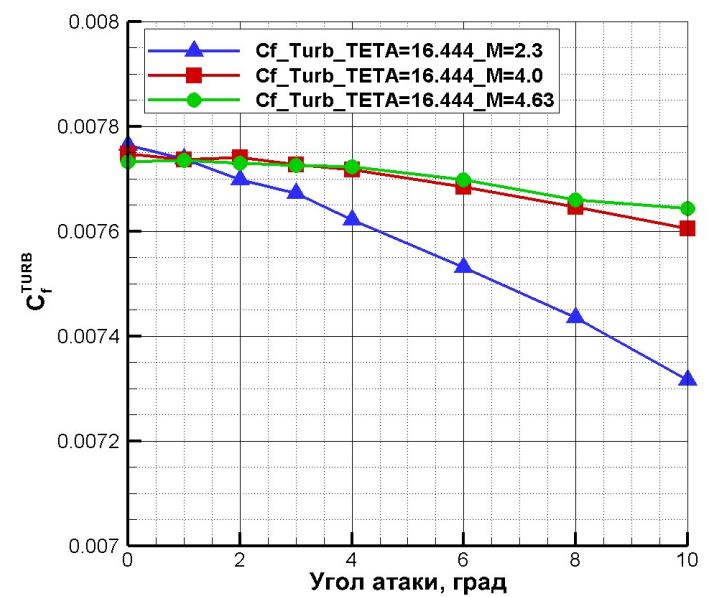

Рис. 28. Коэффициент поверхностного трения для разных чисел Маха при различных углах атаки. Турбулентный случай. $\theta=16.444^{\circ}$

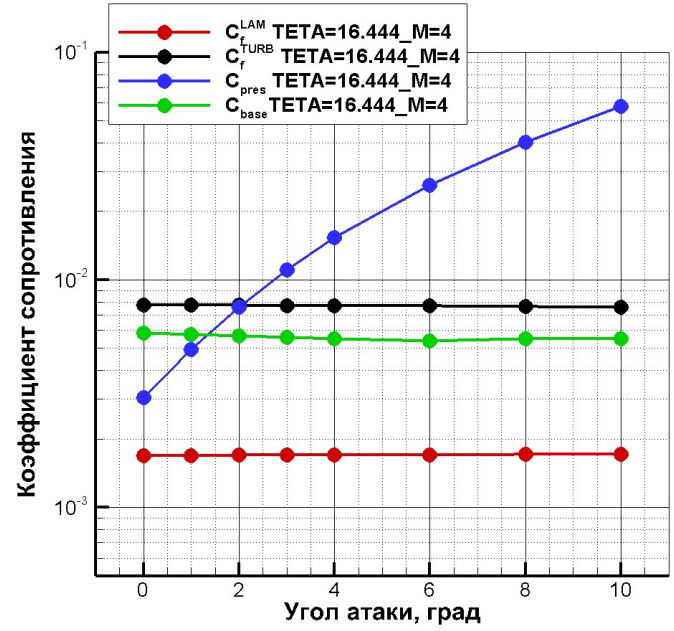

Рис. 30. Компоненты сопротивления волнолета при различных углах атаки. $\mathrm{M}_{\infty}=4, \theta=16.444^{\circ}$

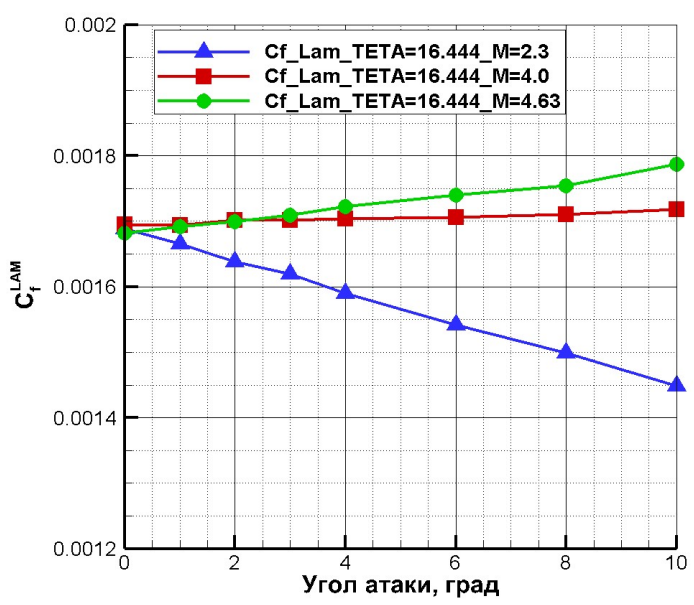

Рис. 27. Коэффициент поверхностного трения для разных чисел Маха при различных углах атаки. Ламинарный случай. $\theta=16.444^{\circ}$

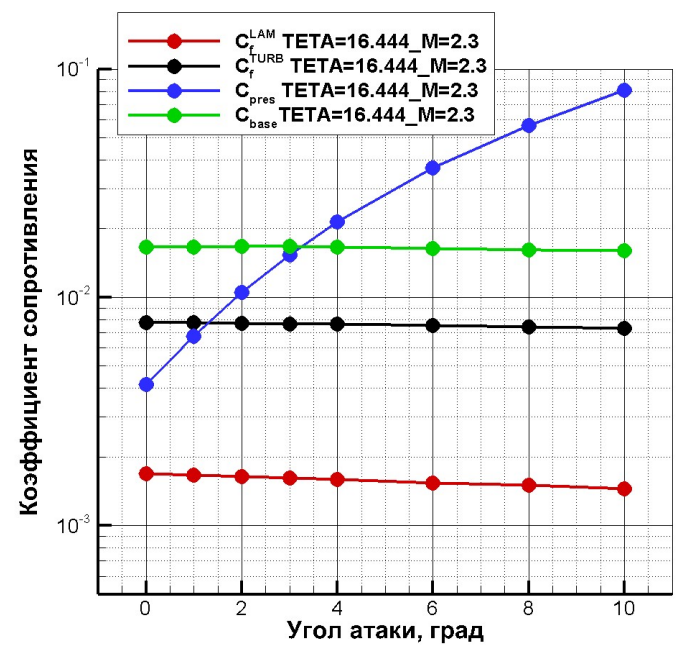

Рис. 29. Компоненты сопротивления ПК-волнолета при различных углах атаки. $\mathrm{M}_{\infty}=2.3$. $\theta=16.444^{\circ}$

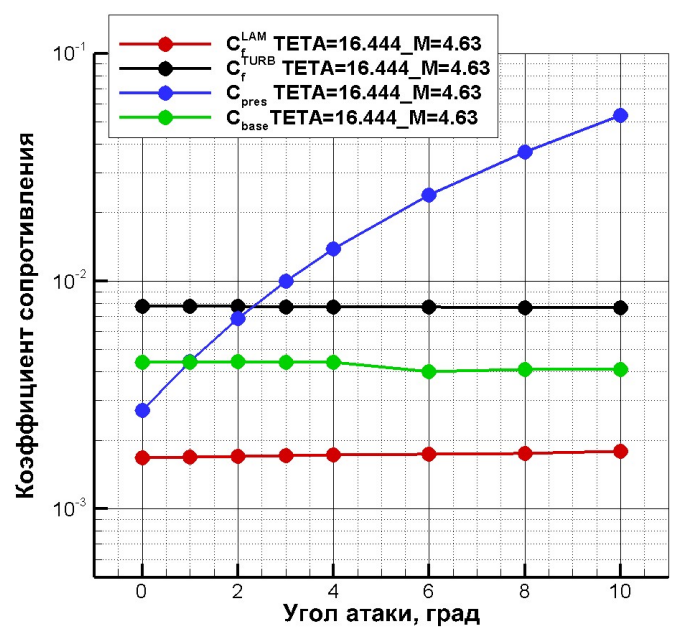

Рис. 31. Компоненты сопротивления волнолета при различных углах атаки. $\mathrm{M}_{\infty}=4.63$. $\theta=16.444^{\circ}$ 


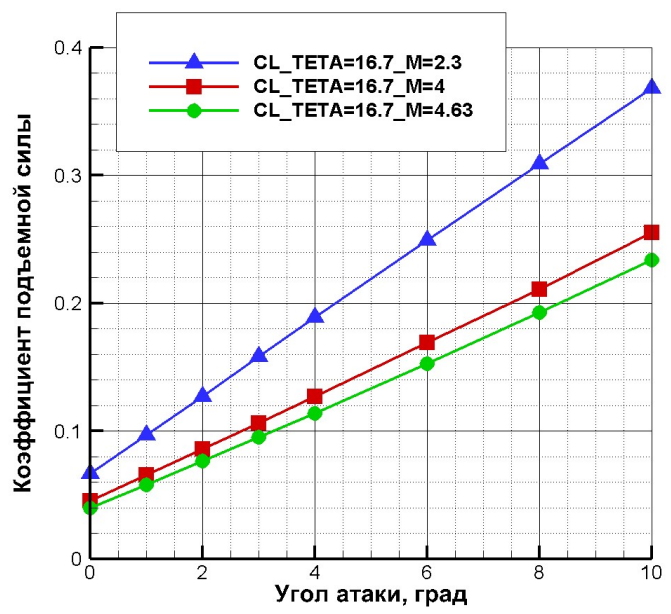

Рис. 32. Коэффициент подъемной силы для разных чисел Маха при различных углах атаки. $\theta=16.7^{\circ}$

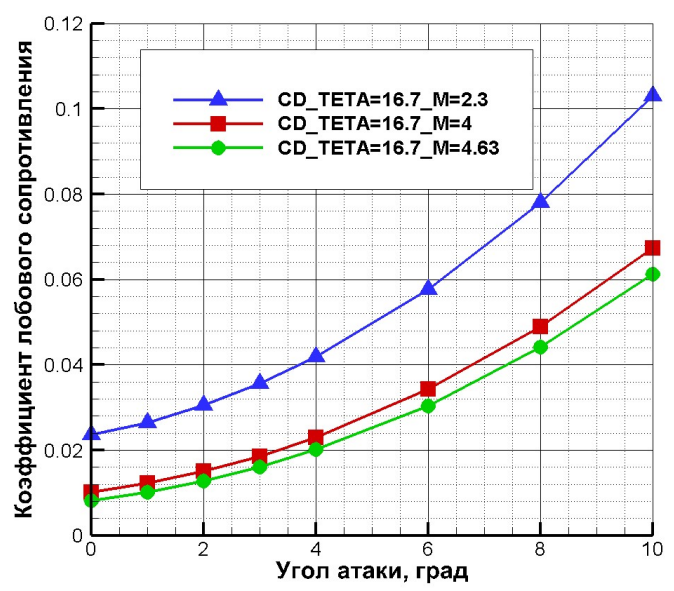

Рис. 34. Коэффициент лобового сопротивления с учетом донного сопротивления для разных чисел Маха при различных углах атаки. $\theta=16.7^{\circ}$

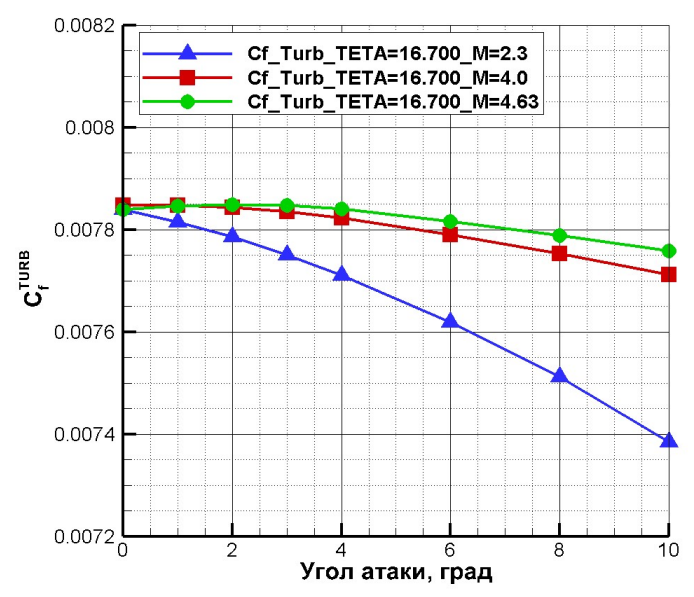

Рис. 36. Коэффициент поверхностного трения для разных чисел Маха при различных углах атаки. Турбулентный случай. $\theta=16.7^{\circ}$

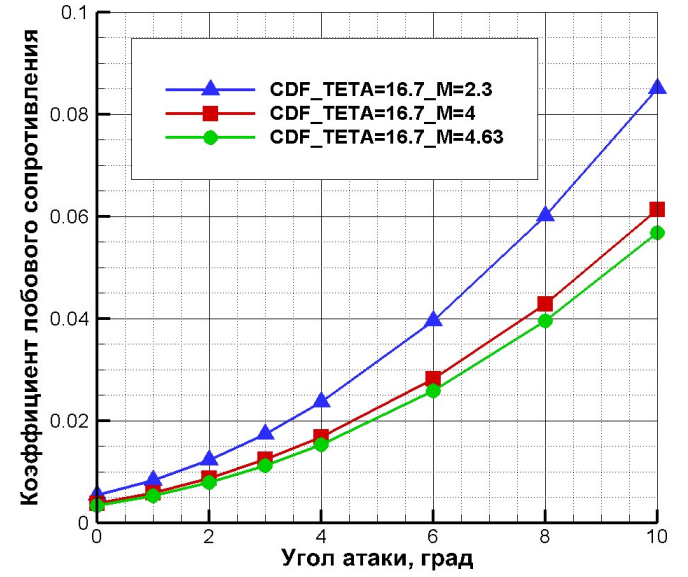

Рис. 33. Коэффициент лобового сопротивления без учета донного сопротивления для разных чисел Маха при различных углах атаки. $\theta=16.7^{\circ}$

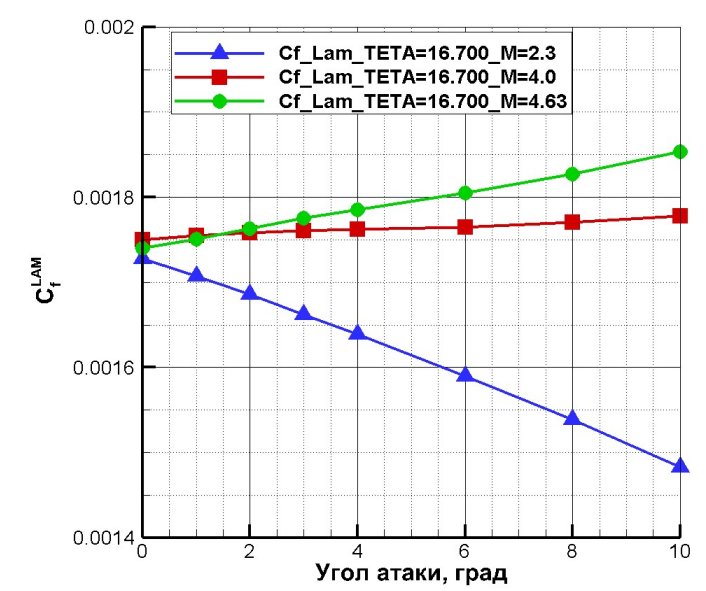

Рис. 35. Коэффициент поверхностного трения для разных чисел Маха при различных углах атаки. Ламинарный случай. $\theta=16.7^{\circ}$

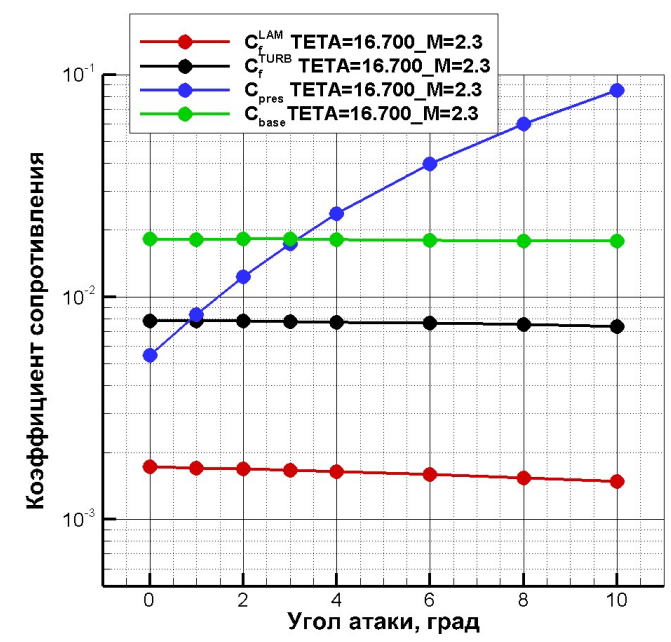

Рис. 37. Компоненты сопротивления ПК-волнолета при различных углах атаки. $\mathrm{M}_{\infty}=2.3$, $\theta=16.7^{\circ}$ 


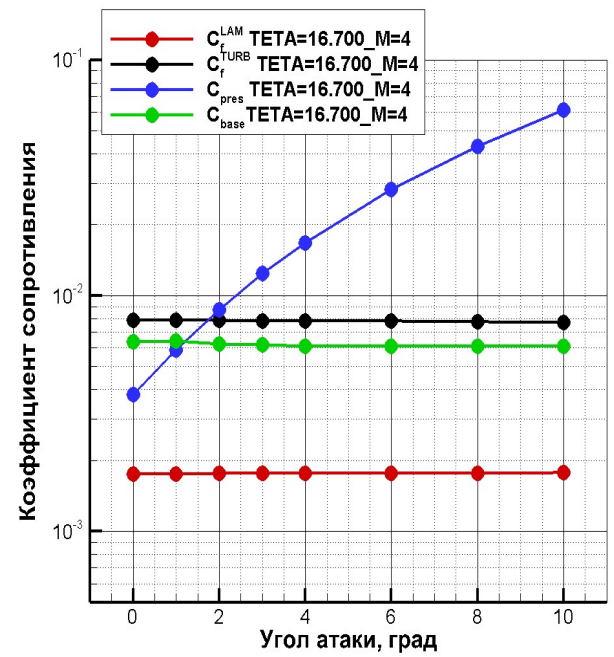

Рис. 38. Компоненты сопротивления ПК-волнолета при различных углах атаки. $\mathrm{M}_{\infty}=4$. $\theta=16.7^{\circ}$

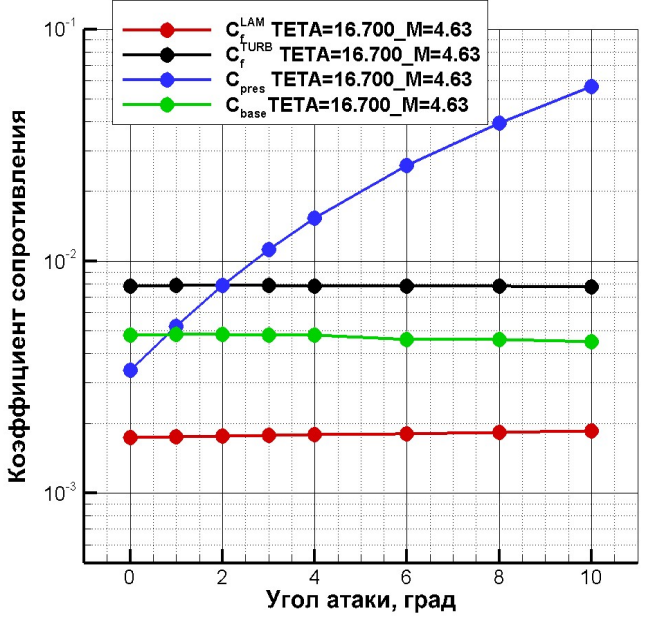

Рис. 39. Компоненты сопротивления ПК-волнолета при различных углах атаки. $\mathrm{M}_{\infty}=4.63$, $\theta=16.7^{\circ}$

На рис. 40-42 демонстрируется характер изменения аэродинамического качества трех моделей ПК-волнолета в зависимости от структуры коэффициента лобового сопротивления. Заключительный этап работы состоял в сопоставлении аэродинамического качества трех моделей, полученного с учетом турбулентного поверхностного трения, с экспериментальными данными. Следует отметить систематическую недооценку искомой аэродинамической характеристики во всем диапазоне исследуемых углов атаки и чисел Маха, что может быть обусловлено завышенными значениями коэффициента лобового сопротивления. В свою очередь причина переоценки данного коэффициента лежит в использовании приближения полностью турбулентного течения при расчете поверхностного трения. Реальный процесс обтекания волнолета сопровождается ламинарно-турбулентным переходом и в этом случае лобовое сопротивление будет ниже, чем при турбулентном обтекании. Рассмотренные модели отличаются прежде всего по площади смачиваемой поверхности, что объясняет разницу в коэффициентах подъемной силы, лобового сопротивления, поверхностного трения. При этом аэродинамическое качество отличается незначительно.

\section{4. Заключение}

В данной статье представлены результаты расчетов аэродинамических характеристик двух типов волнолетов. На примере ЭК-волнолета была выполнена верификация приближенной методики оценки поверхностного трения и проведено сравнение со сторонними расчетными результатами, полученными с использованием уравнений Навье-Стокса, в том числе параболизованных. Далее приближенная методика была применена к ПК-волнолету в части оценки роли сопротивления трения при интерпретации экспериментальных данных. Полученные результаты показывают, что только учет вязкого сопротивления позволяет получить корректные результаты по аэродинамическому качеству в зависимости от числа Маха. Учет донного сопротивления вместо трения приводит к изменению поведения аэродинамического качества по отношению к скорости набегающего потока, что вступает в противоречие с экспериментальными данными. За счет применения турбулентного приближения коэффициент лобового сопротивления получается завышенным, что обуславливает пониженное значение аэродинамического качества по сравнению с экспериментальными данными. 


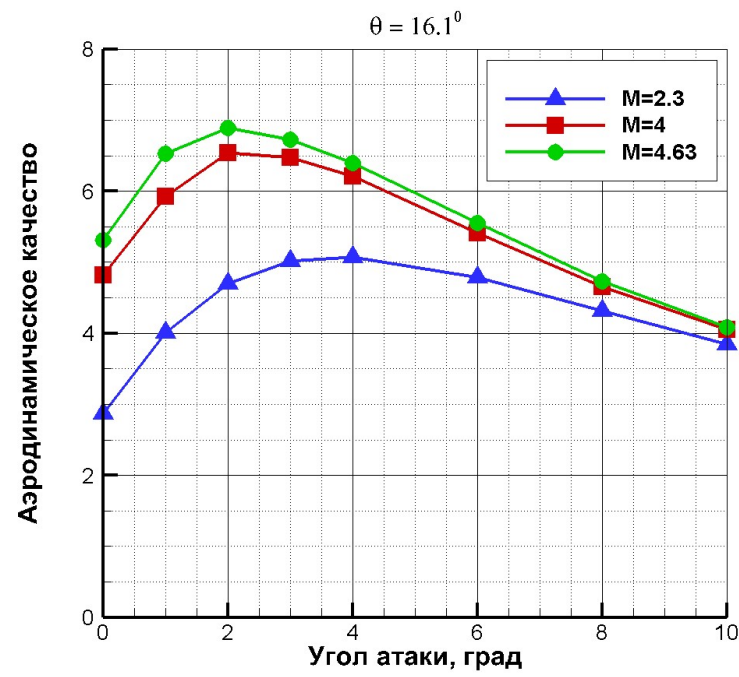

(a)

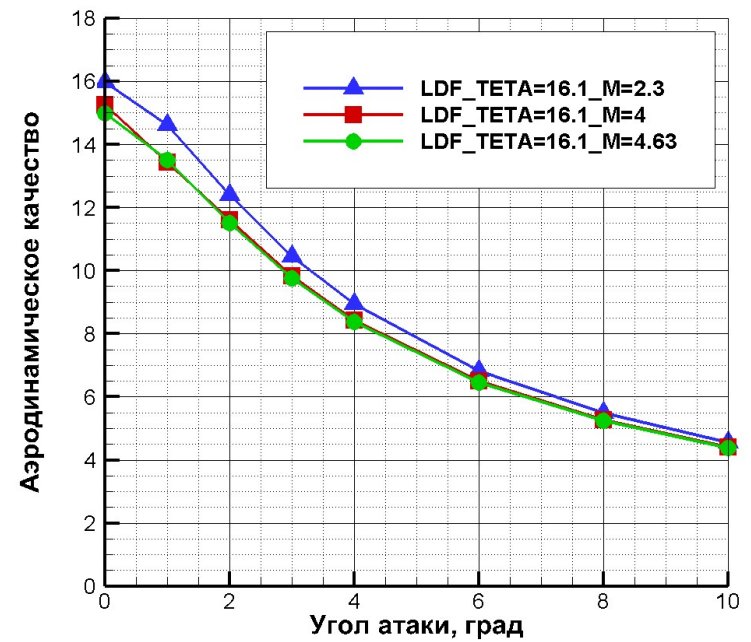

(б)

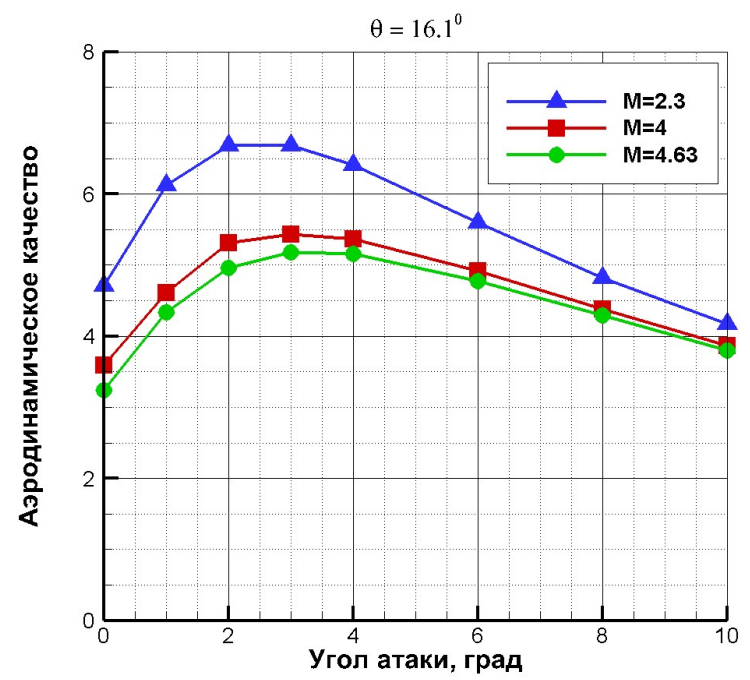

(B)

Рис. 40. Аэродинамическое качество для разных чисел Маха при различных углах атак и при учете в общем сопротивлении ПК-волнолета $(a)$ сопротивления давления и донного сопротивления (б) только сопротивления давления (в) сопротивления давления и турбулентного коэффициента вязкого трения 


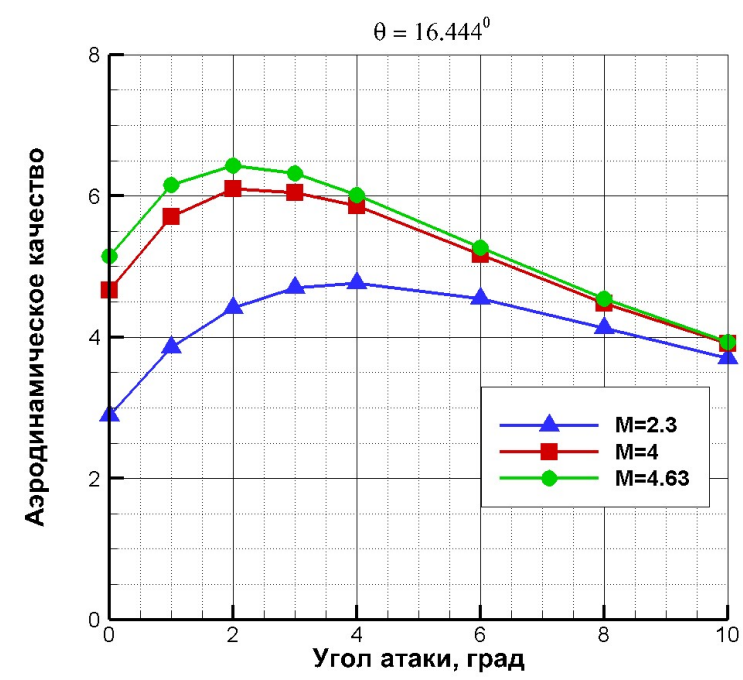

(a)

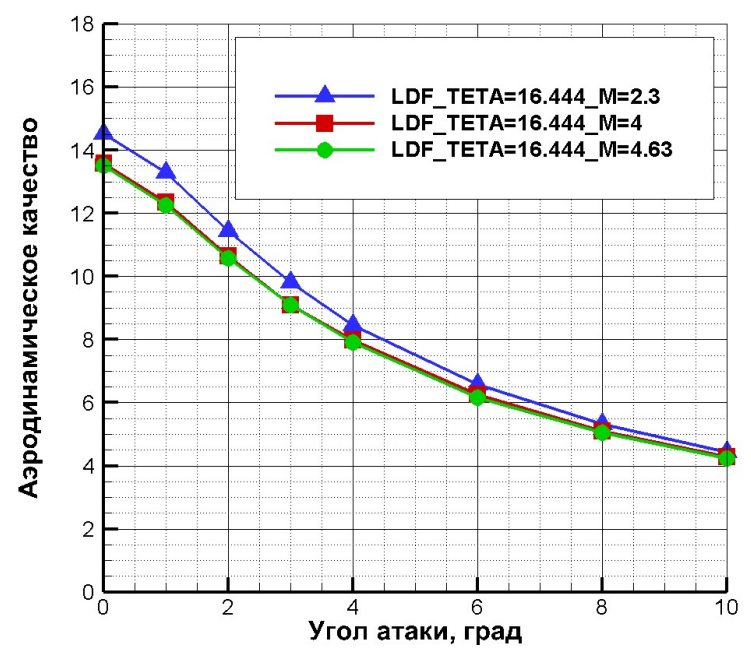

(б)

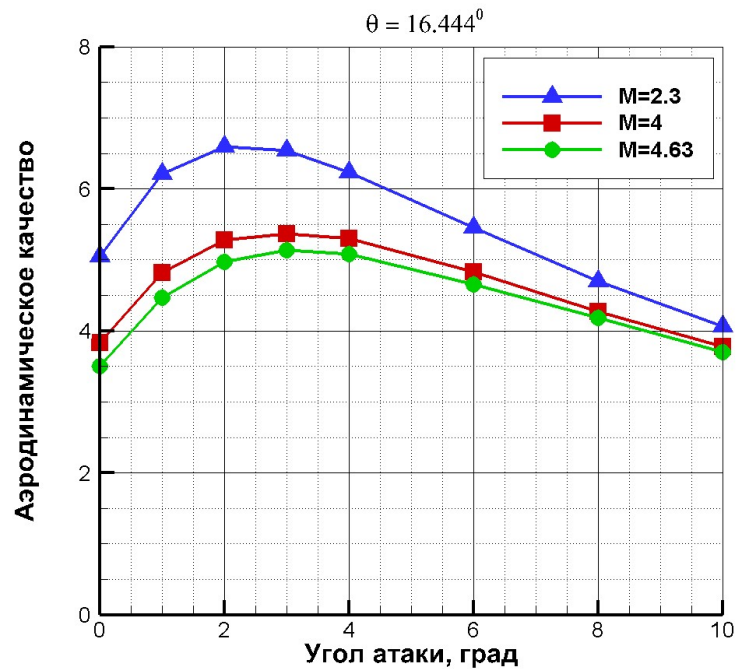

(b)

Рис. 41. Аэродинамическое качество для разных чисел Маха при различных углах атак и при учете в общем сопротивлении ПК-волнолета $(a)$ coпротивления давления и донного сопротивления (б) только сопротивления давления (в) сопротивления давления и турбулентного коэффициента вязкого трения. $\theta=16.444^{\circ}$ 


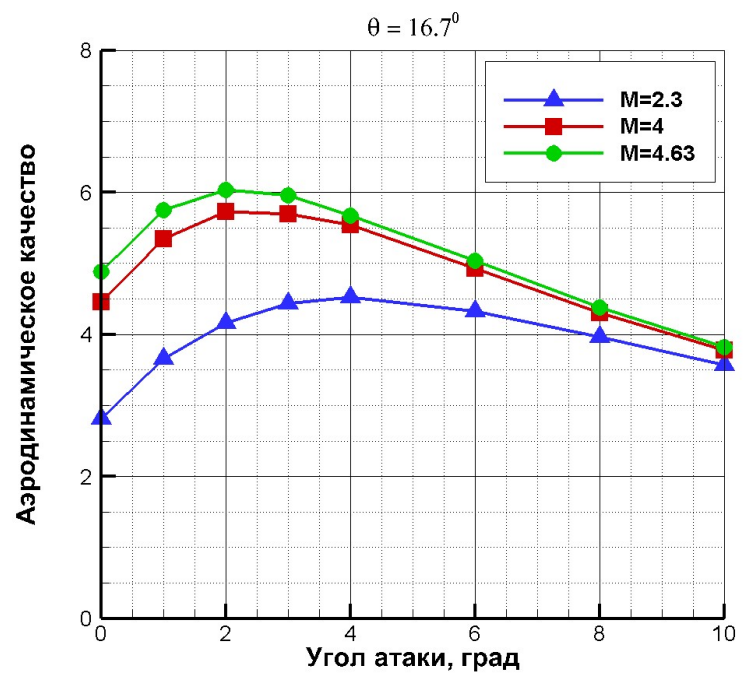

(a)

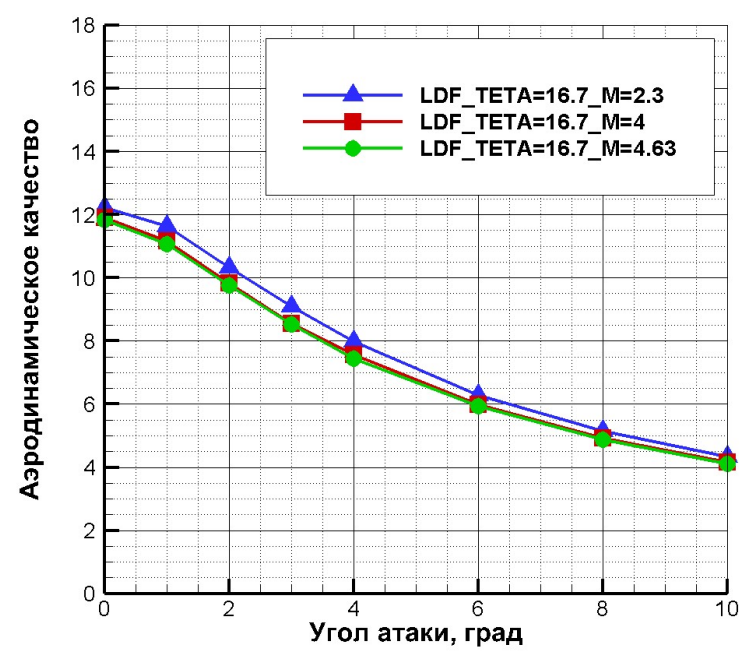

(б)

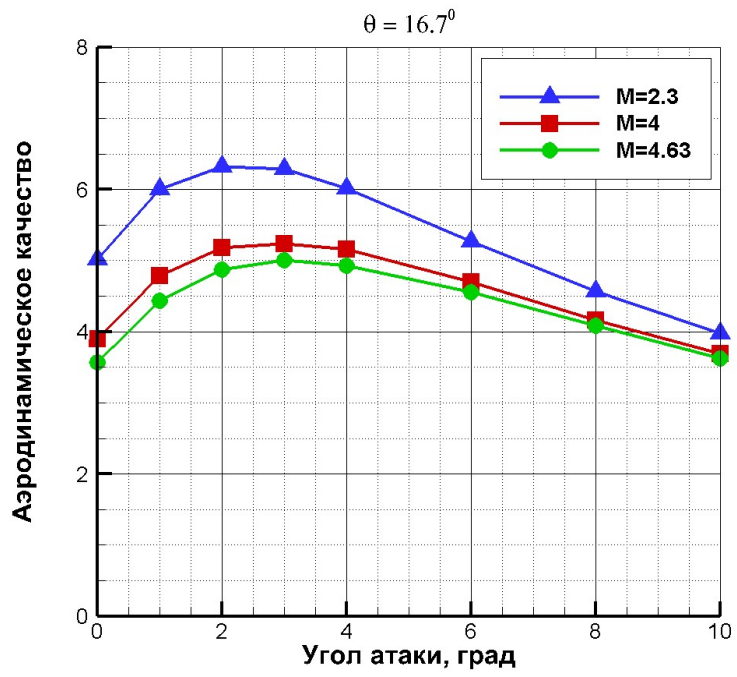

(

Рис. 42. Аэродинамическое качество для разных чисел Маха при различных углах атак и при учете в общем сопротивлении ПК-волнолета $(a)$ сопротивления давления и донного сопротивления (б) только сопротивления давления (в) сопротивления давления и турбулентного коэффициента вязкого трения. $\theta=16.7^{\circ}$ 


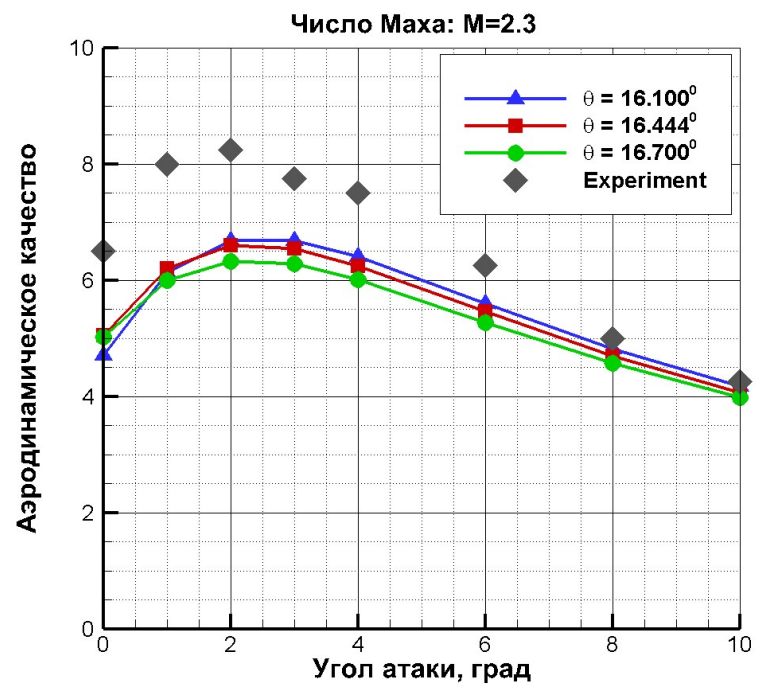

(a)

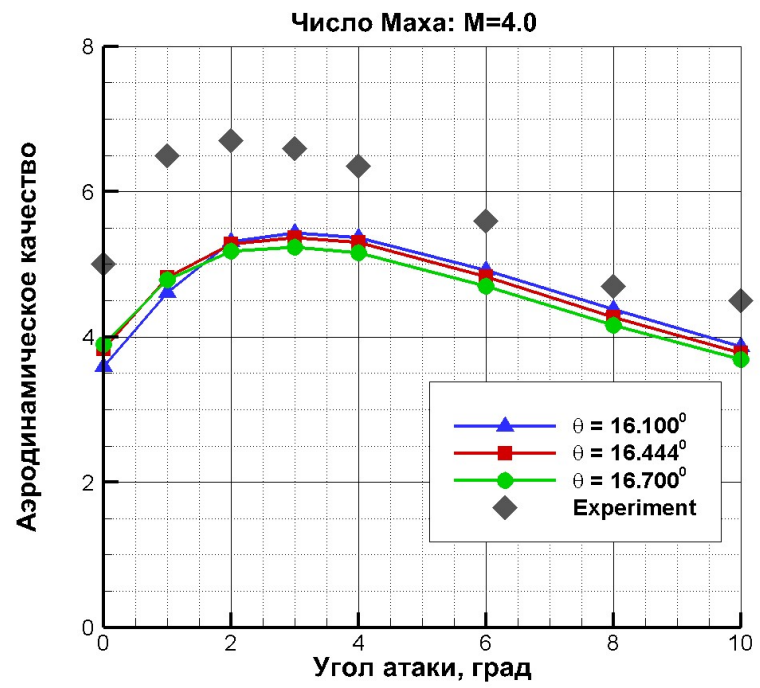

(б)

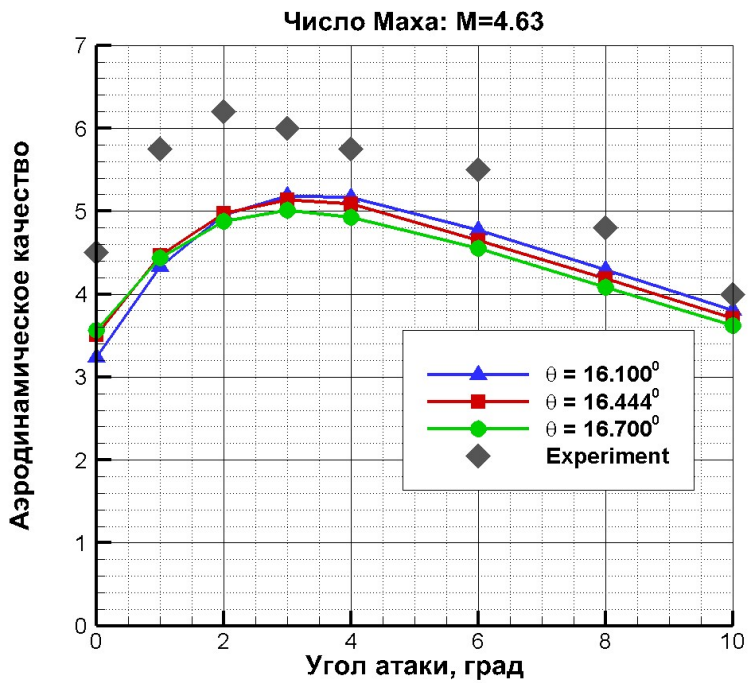

(b)

Рис. 43. Аэродинамическое качество для разных моделей ПК-волнолета при различных углах атак и при учете в общем сопротивлении волнолета сопротивления давления и турбулентного коэффициента вязкого трения в сравнении с экспериментальными данными [22] при (a) $\mathrm{M}_{\infty}=2.3$, (б) $\mathrm{M}_{\infty}=4$, (в) $\mathrm{M}_{\infty}=4.63$ 


\section{Благодарности}

Работа выполнена в рамках государственного задания № AААА-А20-120011690135-5, а также частично поддержана грантом РФФИ 19-01-00515.

\section{Литература}

1. Bowcutt K.G., Anderson J.D., Capriotti D. Viscous Optimized Hypersonic Waveriders // AIAA Paper 87-0272. 1987. pp. 1-19. https://doi.org/10.2514/6.1987-272

2. Corda S., Anderson J.D. Viscous Optimized Hypersonic Waveriders Designed from Axisymmetric Flow Fields // AIAA Paper 88-0369. 1988. pp. 1-14. https://doi.org/10.2514/6.1988-369

3. Кюхеман Д. Аэродинамическое проектирование самолетов. М.: Машиностроение, 1983. 656 с.

4. Liao J.-R., Isaac K.M., Miles J.B., Tsai B.-J. Navier-Stokes Simulation for Cone-Derived Waverider // AIAA Journal. 1992. Vol. 30. No. 6. pp. 1521-1528 https://doi.org/10.2514/3.11096

5. Kato H., Tannehill J.C. Numerical Calculation of Viscous Flow Over Hypersonic Waveriders // AIAA Paper 97-2292. 1997. pp. 1-11 https://doi.org/10.2514/6.1997-2292

6. Bauer S.X.S. Analysis of Two Viscous Optimized Waveriders // Proc. of the $1^{\text {st }}$ Int. Hyper. Waver. Symp. 1990. pp. 1-27.

7. Walz A. Boundary Layers of F1ow and Temperature, 1969, MIT Press

8. White F. Viscous Fluid Flow. McGraw Hill, 1974, pp. 653-657

9. DiCristina V. Three-Dimensional Laminar Boundary Layer Transition on a Sharp $8^{0}$ Cone at Mach 10 // AIAA Journal. Vol. 8. No. 5. 1970, p. 855

10. Harris J.E., Blanchard O.K. Computer Program for Solving Laminar, Transitional and Turbulent Compressible Boundary Layer Equations for Two Dimensional and Axisymnetric Flows //NASA-TM 83207. 1982.

11. Takashima N., Lewis M.J. Navier-Stokes Computation of a Viscous Optimized Waverider // J. Spac. Rock. 1994. V. 31. № 3. pp. 383-391.

12. Anderson J.D., Jr. Hypersonic and high temperature gas dynamics. NY: McGraw-Hill Book Company. 1989

13. Яцухно Д.С. Численное моделирование аэродинамики волнолетов, построенных на скачках уплотнения различной формы//Физико-химическая кинетика в газовой динамике. 2020. Т. 21, вып. 1. http://chemphys.edu.ru/issues/2020-21-1/articles/881/

14. Surzhikov S.T. Validation of computational code UST3D by the example of experimental aerodynamic data // Journal of Physics: Conference Series. 2017. Vol. 815. No. 12023 https://doi.org/10.1088/1742$\underline{6596 / 815 / 1 / 012023}$

15. Surzhikov S.T. Comparative Analysis of the Results of Aerodynamic Calculation of a Spherical Blunted Cone on a Structured and Unstructured Grid // Journal of Physics: Conference Series. 2019. Vol. 1250. No 012007 https://doi.org/10.1088/1742-6596/1250/1/012007

16. Яцухно Д.С., Суржиков С.Т. Метод расщепления по физическим процессам в задаче моделирования обтекания перспективного высокоскоростного летательного аппарата // Вестник МГТУ им. Н.Э. Баумана. Сер. «Машиностроение». 2018. № 1. С. 20-33.

17. Сильвестров П.В., Суржиков С.Т. Расчет аэротермодинамики высокоскоростного летательного аппарата X-43 с использованием компьютерных кодов UST3D и UST3D-AUSMPW//Физикохимическая кинетика в газовой динамике. 2019. Т. 20, вып. 4. http://chemphys.edu.ru/issues/2019$\underline{20-4 / \text { articles } / 865}$

18. Суржиков С.Т. Численная интерпретация экспериментальных данных по аэродинамике модели HB-2 с использованием компьютерных кодов USTFEN и PERAT-3D // Физико-химическая кинетика в газовой динамике. 2020. Т. 21, вып. 1. http://chemphys.edu.ru/issues/2020-21-1/articles/900 
19. Hu Z., Zha G. Simulation of 3D Flows of Propulsion Systems Using an Efficient Low Diffusion E-CUSP Upwind Scheme // AIAA Paper 2004-4082. 2004, pp. 1-12 https://doi.org/10.2514/6.2004$\underline{4082}$

20. Rasmussen M.L. Experimental Forces and Moments on Cone-Derived Waveriders for $\mathrm{M}=3$ to 5 // Journal of Spacecraft and Rockets. 1982. Vol. 19. No. 6. pp. 592-598 https://doi.org/10.2514/3.62306

21. White Frank M. Hypersonic Laminar Viscous Interactions on Inclined Flat Plates // ARS Journal, 32:780-781, 1962.

22. Cockrell Ch.E. Vehicle Integration Effects on Hypersonic Waveriders // NASA-TM-109739. 1994. 132 p. https://ntrs.nasa.gov/archive/nasa/casi.ntrs.nasa.gov/19940029612.pdf

\section{References}

1. Bowcutt, K. G., Anderson, J. D., Capriotti, D., "Viscous Optimized Hypersonic Waveriders," 25th Aerospace Sciences Meeting, AIAA Paper 87-0272, 1987. https://doi.org/10.2514/6.1987-272

2. Corda, S., Anderson, J. D., "Viscous Optimized Hypersonic Waveriders Designed from Axisymmetric Flow Fields," 26th Aerospace Sciences Meeting, Jan. 11-14 Reno Nevada, AIAA Paper 88-0369, 1988. https://doi.org/10.2514/6.1988-369

3. Küchemann, D., The Aerodynamic Design of Aircraft, AIAA education series, 2012, 564 p.

4. Liao, J.-R., Isaac, K. M., Miles, J. B., Tsai, B.-J., "Navier-Stokes Simulation for Cone-Derived Waverider," AIAA Journal, Vol. 30, No. 6, 1992, pp. 1521-1528. https://doi.org/10.2514/3.11096

5. Kato, H., Tannehill, J. C., "Numerical Calculation of Viscous Flow Over Hypersonic Waveriders," 15th Applied Aerodynamics Conference, AIAA Paper 97-2292, June 1997. https://doi.org/10.2514/6.1997-2292

6. Bauer, S. X. S., "Analysis of Two Viscous Optimized Waverider," Proc. of the $1^{\text {st }}$ Int. Hyper. Waver. Symp., 1990, pp. 1-27.

7. Walz, A., Boundary Layers of Flow and Temperature, MIT Press, 1969.

8. White, F., Viscous Fluid Flow, McGraw Hill, 1974, pp. 653-657

9. DiCristina, V., "Three-Dimensional Laminar Boundary Layer Transition on a Sharp $8^{0}$ Cone at Mach 10," AIAA Journal, Vol. 8, No. 5, 1970, p. 855.

10. Harris, J. E., Blanchard, O. K., "Computer Program for Solving Laminar, Transitional and Turbulent Compressible Boundary Layer Equations for Two Dimensional and Axisymnetric Flows," NASA-TM 83207, 1982.

11. Takashima, N., Lewis, M. J., "Navier-Stokes Computation of a Viscous Optimized Waverider," J. Spac. Rock., Vol. 31, No. 3, 1994, pp. 383-391.

12. Anderson, J. D., Jr., Hypersonic and high temperature gas dynamics, NY: McGraw-Hill Book Company, 1989.

13. Yatsukhno, D. S., "Computational Study of the Different Waverider Configurations Aerodynamics," Physical-Chemical Kinetics in Gas Dynamics, Vol.21, No. 1, 2020. http://chemphys.edu.ru/issues/2020-21-1/articles/881/

14. Surzhikov, S. T., "Validation of computational code UST3D by the example of experimental aerodynamic data," Journal of Physics: Conference Series, Vol. 815. No. 12023, 2017. https://doi.org/10.1088/1742-6596/815/1/012023

15. Surzhikov, S. T., "Comparative Analysis of the Results of Aerodynamic Calculation of a Spherical Blunted Cone on a Structured and Unstructured Grid," Journal of Physics: Conference Series, Vol. 1250, No. 012007, 2019.https://doi.org/10.1088/1742-6596/1250/1/012007

16. Yatsukhno, D. S, Surzhikov, S. T., Herald of the Bauman Moscow State Technical University: Mechanical Engineering, No. 1, 2018, pp. 20-33. 
17. Silvestrov, P., Surzhikov, S., "Calculation of aerothermodynamics for high-speed aircraft X-43 using computer code UST3D and UST3D-AUSMPW," Physical-Chemical Kinetics in Gas Dynamics, Vol. 20, No. 4, 2019. http://chemphys.edu.ru/issues/2019-20-4/articles/865

18. Surzhikov, S. T., "Numerical Interpretation of Experimental Data on Aerodynamics of the HB-2 Model Using Computer Codes USTFEN and PERAT-3D," Physical-Chemical Kinetics in Gas Dynamics, Vol. 21, No. 1, 2020. http://chemphys.edu.ru/issues/2020-21-1/articles/900

19. $\mathrm{Hu}, \mathrm{Z}$., Zha, G., "Simulation of 3D Flows of Propulsion Systems Using an Efficient Low Diffusion E-CUSP Upwind Scheme," $40^{\text {th }}$ AIAA/ASME/SAE/ASEE Joint Propulsion Conference and Exhibit, AIAA Paper 2004-4082, 2004, pp. 1-12. https://doi.org/10.2514/6.2004-4082

20. Rasmussen, M. L., "Experimental Forces and Moments on Cone-Derived Waveriders for $\mathrm{M}=3$ to 5," Journal of Spacecraft and Rockets, Vol. 19, No. 6, 1982, pp. 592-598. https://doi.org/10.2514/3.62306

21. White, Frank, M., "Hypersonic Laminar Viscous Interactions on Inclined Flat Plates," ARS Journal, 32:780-781, 1962.

22. Cockrell, Ch. E., "Vehicle Integration Effects on Hypersonic Waveriders," NASA-TM-109739. 1994. 132 p. https://ntrs.nasa.gov/archive/nasa/casi.ntrs.nasa.gov/19940029612.pdf

Статья поступила в редакцию 9 ноября 2020 г. 